$10-1996$

\title{
Semiclassical Scattering in a Circular Semiconductor Microstructure
}

\author{
C. D. Schwieters
}

J. A. Alford

John B. Delos

William \& Mary, jbdelos@wm.edu

Follow this and additional works at: https://scholarworks.wm.edu/aspubs

Part of the Physics Commons

\section{Recommended Citation}

Schwieters, C. D.; Alford, J. A.; and Delos, John B., Semiclassical Scattering in a Circular Semiconductor Microstructure (1996). Physical Review B, 54(15), 652-688.

https://doi.org/10.1103/PhysRevB.54.10652

This Article is brought to you for free and open access by the Arts and Sciences at W\&M ScholarWorks. It has been accepted for inclusion in Arts \& Sciences Articles by an authorized administrator of W\&M ScholarWorks. For more information, please contact scholarworks@wm.edu. 


\title{
Semiclassical scattering in a circular semiconductor microstructure
}

\author{
C. D. Schwieters, J. A. Alford, and J. B. Delos \\ Physics Department, The College of William and Mary, Williamsburg, Virginia 23187; \\ Joint Institute for Laboratory Astrophysics, University of Colorado, Boulder, Colorado 80309; \\ and National Institute of Standards and Technology, Boulder, Colorado 80309
}

(29 February 1996; revised manuscript received 29 May 1996)

\begin{abstract}
The conductance of a microscopic junction shows fluctuations caused by quantum interference of waves that follow different paths between the leads. We give a semiclassical formula for these fluctuations. The theory utilizes trajectories which travel between the centers of the lead apertures; it also incorporates diffraction at these apertures. We extend the theory to include "ghost paths," which scatter diffractively off the lead mouths. Semiclassical $S$-matrix elements are computed for a circular junction over a range of Fermi wave numbers, and the large-scale structure of these matrix elements shows good agreement with quantum results. Finally, we propose a hypothesis about the effect of the quantum coherence length on the $S$ matrix and on the semiclassical sum. [S0163-1829(96)03935-5]
\end{abstract}

\section{INTRODUCTION}

Quantum transport of electrons through semiconductor microjunctions has been observed in recent experiments. ${ }^{1,2}$ Micron-size two-dimensional junctions have been made with such purity that both the quantum coherence length and the mean free path for elastic collisions of electrons with defects are large compared to the size of the junction. In these experiments conditions are such that the electrons can be described as a two-dimensional ideal Fermi gas of noninteracting particles. In these circumstances a classical electron would bounce ballistically through the cavity; in quantum mechanics the electron wave function scatters elastically from the walls of the junction. This behavior is shown schematically in Fig. 1.

The conductance of such junctions has been measured, ${ }^{1,2}$ and has been found to oscillate strongly as the Fermi energy or the strength of an imposed magnetic field is varied. Statistical properties of these fluctuations have been studied, and compared with predictions from random matrix theory. ${ }^{3-6}$ Statistical properties of the fluctuations are similar to those arising from phase-coherent transport through disordered systems. ${ }^{7}$ These studies have led to an understanding of statistical characteristics common to all generic mesoscopic conductors. Also, the high-frequency part of the power spectrum has been studied, and its structure (power-law decay for regular systems vs exponential decay for chaotic systems) has been compared with predictions from general semiclassical arguments. ${ }^{8,9}$

Statistical properties of fluctuations may be interesting, but it is appropriate now to ask more detailed questions. Can the conductance fluctuations themselves be predicted? On the experimental side, will junctions be created such that the quantum fluctuations of conductance are reliably reproducible, and depend only on the lithographically observed geometry of the junction? On the theoretical side, can we develop simple formulas or algorithms that predict the fluctuations of conductance vs Fermi energy or vs magnetic field? Toward this end a number of groups have performed exact quantum calculations on representative systems, ${ }^{10-15}$ and found the same qualitative behavior as found in experiment: rapid oscillations of the resistance as one changes the energy of the electrons or the strength of the magnetic field. In this paper we derive simple semiclassical formulas that predict some of the large-scale structure of conductance fluctuations.

The essence of the theory is very simple (Fig. 1). Electron waves approach the junction in one of the leads, and, where the lead joins the junction, the waves diffract into the enclosure. Diffraction creates a circular wave outgoing from the entrance lead. This wave bounces around the enclosure following classical paths; the phase of the wave is the classical action on each path, and the amplitude of the wave is the square root of the classical density. Each classical path from entrance to exit contributes a term to the wave function near the exit. These waves diffract out the exit, giving a transmitted current. Interference of waves from various paths pro-

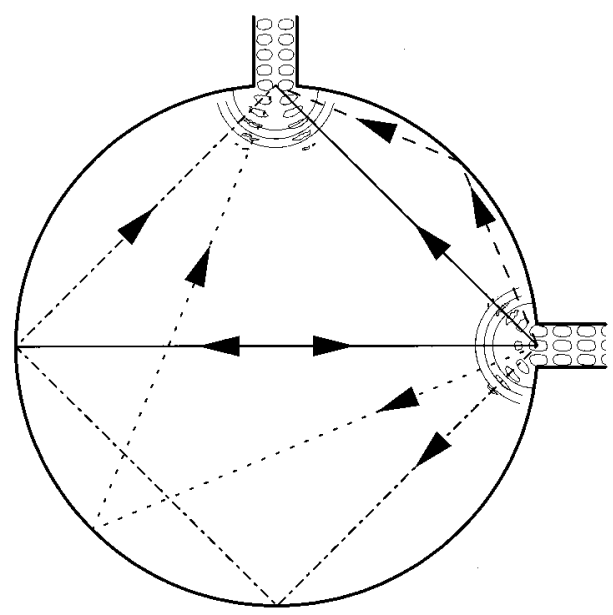

FIG. 1. Overview of the semiclassical method. A quantum wave incoming from a mode of the entrance lead diffracts as it enters the junction, and then travels along classical paths inside the junction. At the exit each trajectory contributes a term to the flux that goes out into each mode of the exit lead. 
duces oscillations in the transmitted current.

The important assumptions involved in the theory are that the de Broglie wavelength is small compared to the size of the junction, and that the widths of the leads are small compared to the size of the junction. The shape of the junction is not important; for this first paper we consider a circular junction with two leads attached at right angles (we show some results for a stadium-shaped junction in Appendix IV D). We use hard-wall boundary conditions; soft-wall boundary conditions are easy to incorporate into a semiclassical theory. Implicitly, we presume that the lithographic shapes of junctions can be taken seriously: the interference pattern results from the visible geometry of the device, not from invisible defects.

Several previous studies of ballistic transport have incorporated semiclassical concepts in some way. Jalabert, Baranger and Stone ${ }^{11}$ and Lin and Jensen ${ }^{16}$ considered interfering paths, but did not consider diffraction. Other groups have applied periodic orbit concepts to the problem of the conductance of periodic arrays of antidots. ${ }^{17,18}$

In the current paper, we treat diffraction within the Kirchhoff approximation. The geometric theory of diffraction (GTD) (Ref. 19) provides an alternate method to approximate the effects of diffraction using additional (nongeometric) trajectories. In recent work the GTD has been successfully used to account for some effects of diffraction in simple open systems. $^{20}$

Like much previous work, the present study relies on the pioneering work of Landauer ${ }^{21}$ and Büttiker. ${ }^{22}$ The primary contribution of the present work is to incorporate the effects of diffraction. This enables us to perform calculations in an energy regime where comparison with quantum-mechanical results is feasible. In addition, in the quantum framework, we improve upon previous computational methods; this was necessary to obtain reliable comparisons between semiclassical and quantum calculations.

\section{CONNECTION OF CONDUCTANCE TO SCATTERING THEORY}

Through the work of Landauer ${ }^{21}$ and others, ${ }^{22,23}$ it has been established that $g_{j i}$, the conductance between leads $i$ and $j$, connected to a mesoscopic conductor, is related to the associated quantum scattering problem through the "corrected Landauer formula." For each spin degree of freedom,

$$
g_{j i}=\frac{e^{2}}{h} \sum_{n, m}\left|t_{m n}^{(j i)}\right|^{2},
$$

where $t_{m n}^{(j i)}$ is the transmission amplitude from transverse mode $n$ of the entrance lead to mode $m$ of the exit lead, and the sum is over all open modes. These transmission amplitudes are elements of the $S$ matrix, $S_{m n}^{(j i)}$, for which $j \neq i$.

Typically, the leads of these mesoscopic semiconductor junctions contain just a few transverse modes; however, the junction itself can be many wavelengths across. In these circumstances, the leads are best described using quantum mechanics, while the wave function inside the junction can be represented using semiclassical methods. The two descriptions must therefore be connected at the mouths of the leads. The geometry of this aperture is important, ${ }^{10}$ as is illustrated

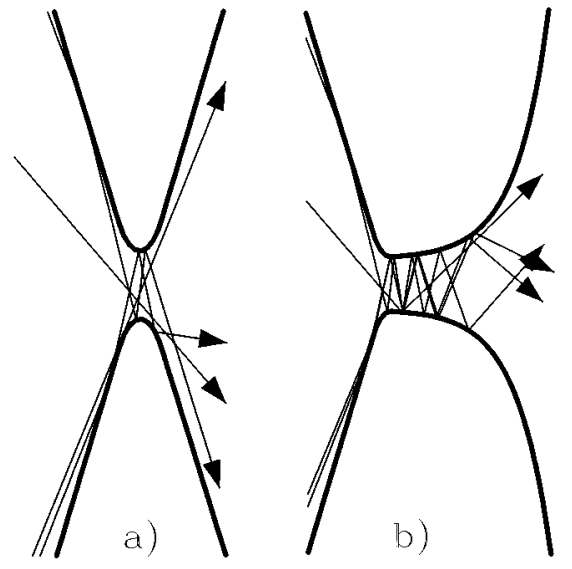

FIG. 2. Different entrance geometries. A short, narrow entrance would allow electrons to pass through from almost any angle, while a horn-shaped aperture would give a more collimated beam (Ref. 10).

in Fig. 2. In Fig. 2(a) the aperture is a pinched constriction between the junction and the electron reservoir; in this case transverse lead modes are not well defined, and electrons can enter the junction at almost any angle. By contrast, Fig. 2(b) depicts a horn geometry, in which the entering trajectories are more collimated in the forward direction. In general, if the width of the aperture is comparable to the de Broglie wavelength, diffraction should be incorporated when connecting the quantum lead wave function to a semiclassical junction wave function.

For concreteness we specify a particular entrance geometry with perfect linear leads in Fig. 3. (A different entrance geometry was addressed in Ref. 24.) Here we take the walls of the leads and the junction walls as infinitely hard, so that the wave function goes to zero at the walls. In real systems, the potential energy representing the walls is continuous, but,

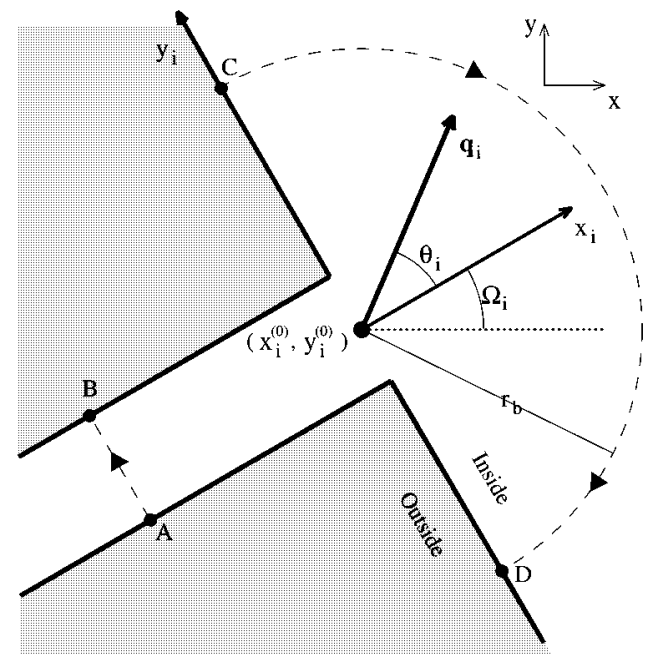

FIG. 3. The entrance geometry of the wire lead and definition of local coordinates. Lead coordinates $\left(x_{i}, y_{i}\right)$ have origin at the center of the mouth of the $i$ th lead, and are rotated by $\Omega_{i}$ relative to space-fixed coordinates. Each point $\left(x_{i}, y_{i}\right)$ can also be described by polar coordinates $\left(r_{i}, \theta_{i}\right)$. The flux integral around the closed curve $A B C D A$ vanishes. 
in the absence of a definitive experimental wall potential, we use the current simplifying assumption. In addition, we will take the magnetic field to be zero in the present derivation.

\section{SEMICLASSICAL FORMULA}

\section{A. States in the leads}

A "space-fixed frame" is defined for the whole system such that $(x, y)$ represents the position of the electron; for a circular junction the origin of these coordinates is at the center of the circle. Local coordinates at the $i$ th lead are defined in Fig. 3. The points $\left(x=x_{i}^{(0)}, y=y_{i}^{(0)}\right)$ are the center of the end of the $i$ th lead; lead coordinates $\left(x_{i}, y_{i}\right)$ are translated to this origin and rotated, so $y_{i}$ is across the lead and $x_{i}$ is along the lead pointing into the junction. The relationship between the lead coordinates $\left[\mathbf{q}_{i}=\left(x_{i}, y_{i}\right)\right]$ and junction-centered coordinates $[\mathbf{q}=(x, y)]$ is given by

$$
\left(\begin{array}{l}
x \\
y
\end{array}\right)=\left(\begin{array}{c}
x_{i}^{(0)} \\
y_{i}^{(0)}
\end{array}\right)+\left(\begin{array}{cc}
\cos \Omega_{i} & -\sin \Omega_{i} \\
\sin \Omega_{i} & \cos \Omega_{i}
\end{array}\right)\left(\begin{array}{c}
x_{i} \\
y_{i}
\end{array}\right) .
$$

The flux-normalized incoming wave function in the $i$ th lead is

$$
\psi_{i, n}^{\text {lead,in }}\left(\mathbf{q}_{i}\right)=\frac{1}{\sqrt{v_{i, n}}} e^{i k_{i, n} x_{i}} \phi_{i, n}\left(y_{i}\right),
$$

and zero outside of the lead. Here $k_{i, n}=\sqrt{k_{F}^{2}-\left(n \pi / w_{i}\right)^{2}}$ is the longitudinal component of the wave vector for mode $n$ in lead $i$, with the corresponding velocity $v_{i, n}=k_{i, n} \hbar / M . M$ is the effective mass of the electron, taken as 1 in our calculations, and $w_{i}$ is the lead width. The transverse component of the wave function, $\phi_{i, n}\left(y_{i}\right)$, is the solution to the onedimensional particle-in-a-box for our hard-wall boundary conditions:

$$
\phi_{i, n}\left(y_{i}\right)=\left(\frac{2}{w_{i}}\right)^{1 / 2} \sin \left[n \pi\left(\frac{y_{i}}{w_{i}}+\frac{1}{2}\right)\right] .
$$

The corresponding outgoing state $\psi_{j, m}^{\text {lead,out }}$ for the $m$ th mode of the $j$ th lead is given by the complex conjugate of Eq. (3), with the appropriate change of labels, $(i, n) \rightarrow(j, m)$ :

$$
\psi_{j, m}^{\text {lead,out }}\left(\mathbf{q}_{j}\right)=\frac{1}{\sqrt{v_{j, m}}} e^{-i k_{j, m} x_{j}} \phi_{j, m}\left(y_{j}\right) .
$$

\section{B. Boundary conditions and definition of the $S$ matrix}

Suppose that electrons approach the junction only in the $n$th mode of the $i$ th lead. Where the lead meets the junction, the wave diffracts, bounces around inside the junction, and then electrons leave the junction from every lead in all energetically allowed modes. The wave function representing this physical situation is called $\psi_{i, n}^{+}(\mathbf{q})$. The $S$ matrix specifies the amplitude for finding the electron in the outgoing modes of any lead. In the asymptotic region (far enough so that closed channels do not contribute) of lead $j$, this wave function is

$$
\psi_{i, n}^{+}(\mathbf{q})=\delta_{j i} \psi_{i, n}^{\text {lead,in }}\left(\mathbf{q}_{i}\right)+\sum_{m=1}^{M_{j}} S_{m n}^{(j i)} \psi_{j, m}^{\text {lead,out }}\left(\mathbf{q}_{j}\right),
$$

where $M_{j}$ is the highest open lead mode.

$S$-matrix elements are of course related to the currents in the various leads. We define the current density operator in the usual way ${ }^{25}$

$$
\mathfrak{J}(\mathbf{Q})=\left(\frac{1}{2}\right)\left[\mathbf{v}_{\mathrm{op}} \delta(\mathbf{q}-\mathbf{Q})+\delta(\mathbf{q}-\mathbf{Q}) \mathbf{v}_{\mathrm{op}}\right]
$$

where $\mathbf{v}_{\text {op }}$ is the velocity operator [equal to $-i(\hbar / M) \nabla$ in the absence of magnetic fields].

Let us draw a boundary across the $j$ th lead in the asymptotic region, and let the collection of points $\{\mathbf{Q}\}$ be the points on this boundary (Fig. 3),

$$
\left\{\mathbf{Q}_{j}\right\}=\left\{x_{j}=x_{b},-\frac{w}{2} \leqslant y_{j} \leqslant \frac{w}{2}\right\} .
$$

Then we define the current operator $\mathfrak{J}_{j}$ for the $j$ th lead as

$$
\mathfrak{J}_{j}=\int_{-w / 2}^{w / 2} d y_{j} \mathfrak{J}\left(x_{j}=x_{b}, y_{j}\right) \cdot \hat{\mathbf{n}}_{j},
$$

where $\hat{\mathbf{n}}_{j}=-\hat{\mathbf{x}}_{j}$ is the outward-pointing normal. The $S$-matrix element $S_{m n}^{(j i)}$ is equal to a matrix element of this operator

$$
\begin{aligned}
S_{m n}^{(j i)}= & \left\langle\psi_{j, m}^{\text {lead,out }}\left|\mathfrak{J}_{j}\right| \psi_{i, n}^{+}\right\rangle \\
= & \frac{i \hbar}{2 M} \int_{-w / 2}^{w / 2} d y_{j}\left[\left(\psi_{j, m}^{\text {lead,out }}\left(x_{b}, y_{j}\right)\right)^{*} \frac{\partial}{\partial x_{j}} \psi_{i, n}^{+}\left(x_{b}, y_{j}\right)\right. \\
& \left.-\psi_{i, n}^{+}\left(x_{b}, y_{j}\right) \frac{\partial}{\partial x_{j}}\left(\psi_{j, m}^{\text {lead,out }}\left(x_{b}, y_{j}\right)\right)^{*}\right] .
\end{aligned}
$$

[Proof: substitute Eqs. (6) and (9) into (10a).]

The same formula holds if the boundary of chosen differently. In general, if $\psi_{1}(\mathbf{q})$ and $\psi_{2}(\mathbf{q})$ are any two exact solutions to the stationary Schrödinger equation, $\mathcal{C}$ is any closed contour, and $\hat{\mathbf{n}}$ the outward-pointing normal on this curve, then by Green's theorem a flux-type integral around the curve must vanish,

$$
\oint_{\mathcal{C}} d s\left[\psi_{1}^{*}(\mathbf{q}) \boldsymbol{\nabla} \psi_{2}(\mathbf{q})-\psi_{2}(\mathbf{q}) \boldsymbol{\nabla} \psi_{1}^{*}(\mathbf{q})\right] \cdot \hat{\mathbf{n}}=0 .
$$

For example, the integral around the closed mushroomshaped curve in Fig. 3 vanishes, and since the wave functions vanish at the walls, it follows that the integral across the lead $(A B)$ is equal to the integral around the semicircle $(D C)$ at radius $r_{b}$, or

$$
\begin{aligned}
S_{m n}^{(j i)}= & \frac{i \hbar}{2 M} \int_{-\pi / 2}^{\pi / 2} r_{b} d \theta_{j}\left[\left(\psi_{j, m}^{\text {out }}\left(r_{b}, \theta_{j}\right)\right)^{*} \frac{\partial}{\partial r_{j}} \psi_{i, n}^{+}\left(r_{b}, \theta_{j}\right)\right. \\
& \left.-\psi_{i, n}^{+}\left(r_{b}, \theta_{j}\right) \frac{\partial}{\partial r_{j}}\left(\psi_{j, m}^{\text {out }}\left(r_{b}, \theta_{j}\right)\right)^{*}\right] .
\end{aligned}
$$

This is the formula we will use for evaluation of $S$-matrix elements. However, since the required lead state [Eq. (5)] is only defined within lead $j$, we cannot use it to 
evaluate $\psi_{j, m}^{\text {out }}\left(r_{b}, \theta_{j}\right)$ on the circular boundary: we must use an appropriate continuation of Eq. (5) into the junction.

\section{Diffraction at the entrance aperture}

When the incoming wave enters the junction, it diffracts. We will approximate this process as Kirchhoff diffraction ${ }^{26}$ from the lead into an infinite half-plane. The Kirchhoff formula for this situation is derived from the KirchhoffHelmholtz equation

$$
\psi(\mathbf{q})=\oint d s^{\prime}\left[\psi\left(\mathbf{q}^{\prime}\right) \boldsymbol{\nabla}^{\prime} G\left(\mathbf{q}, \mathbf{q}^{\prime}\right)-G\left(\mathbf{q}, \mathbf{q}^{\prime}\right) \boldsymbol{\nabla}^{\prime} \psi\left(\mathbf{q}^{\prime}\right)\right] \cdot \hat{\mathbf{n}}^{\prime}
$$

Here $\mathbf{q}$ is an arbitrary point inside the junction, and the integration contour is any closed boundary surrounding q. $\boldsymbol{\nabla}^{\prime}$ denotes the derivative with respect to the primed (boundary) coordinates, and $\hat{\mathbf{n}}^{\prime}$ is a unit vector pointing inward from the boundary. Equation (13) is exact. In the Kirchhoff approximation we take the boundary to be the line segment $\left(x_{j}{ }^{\prime}=0,-w / 2<y_{j}{ }^{\prime}<w / 2\right)$ defining the boundary between the $j$ th lead and the junction, and on the right-hand side of Eq. (13) we take $\psi\left(\mathbf{q}^{\prime}\right)$ to be the incoming lead wave function $\psi_{i, n}^{\text {lead,in }}$ defined in Eq. (3).

$G\left(\mathbf{q}, \mathbf{q}^{\prime}\right)$ is taken to be the two-dimensional free-particle Green function, which is ${ }^{27}$

$$
G\left(\mathbf{q}, \mathbf{q}^{\prime}\right)=\frac{i}{4} H_{0}^{(1)}\left(k_{F}\left|\mathbf{q}-\mathbf{q}^{\prime}\right|\right)
$$

where $H_{0}^{(1)}(x)$ is the Hankel function of the first kind. The Green function and its derivative required in Eq. (13) are approximated for large $\left|\mathbf{q}-\mathbf{q}^{\prime}\right|, \mathbf{q}^{\prime}$ small, by

$$
\begin{gathered}
G\left(\mathbf{q}, \mathbf{q}^{\prime}\right) \approx \frac{1}{\sqrt{8 \pi k_{F} r}} e^{i k_{F} r-i k_{F} r^{\prime} \cos \left(\theta-\theta^{\prime}\right)+i \pi / 4} \\
\frac{\partial}{\partial x^{\prime}} G\left(\mathbf{q}, \mathbf{q}^{\prime}\right) \approx\left(\frac{k_{F}}{8 \pi r}\right)^{1 / 2} \cos (\theta) e^{i k_{F} r-i k_{F} r^{\prime} \cos \left(\theta-\theta^{\prime}\right)-i \pi / 4}
\end{gathered}
$$

The left-hand side of Eq. (13), $\psi(\mathbf{q}) \equiv \psi_{i, n}^{\text {in,diff }}(\mathbf{q})$, is then the appropriate wave function diffracted into the junction from the $n$th incoming mode of the $i$ th lead. Inserting Eqs. (3) and (15) into Eq. (13) and integrating only over the lead mouth, we obtain

$$
\psi_{i, n}^{\mathrm{in}, \mathrm{diff}}\left(\mathbf{q}_{i}\right) \approx \frac{e^{i k_{F} r_{i}}}{\sqrt{r_{i}}} \Theta_{i, n}^{\mathrm{in}}\left(\theta_{i}\right)
$$

where

$$
\begin{aligned}
\Theta_{i, n}^{\text {in }}\left(\theta_{i}\right)= & -n \sqrt{\pi w_{i}}\left(\frac{k_{F}}{v_{i, n}}\right)^{1 / 2} e^{i \pi / 4}\left(\cos \left(\theta_{i}\right)+k_{i, n} / k_{F}\right) \\
& \times \chi_{n}\left(\frac{w_{i} k_{F}}{2} \sin \theta_{i}\right), \\
\chi_{n}(a)= & {\left[(n \pi)^{2}-(2 a)^{2}\right]^{-1} \begin{cases}i \cos (a), & n \text { odd } \\
-\sin (a), & n \text { even. }\end{cases} }
\end{aligned}
$$

In Eq. (16) we see a circular wave propagating radially outward from the entrance lead into the junction with an angular dependence given by $\Theta_{i, n}^{\text {in }}\left(\theta_{i}\right)$. As seen in Fig. 4, this angular component is peaked near $\sin \theta_{i}= \pm n \pi /\left(w_{i} k_{F}\right)$, the angles for a classical electron with transverse and longitudinal momenta $n \pi \hbar / w_{i}$ and $k_{i, n} \hbar$, respectively. The angular dependence becomes strongly peaked around the classical angles for large wave vector, as one would expect. Near threshold $\left(k_{F}=n \pi / w_{i}\right)$ this formula breaks down. It no longer approximately obeys the boundary conditions $\psi=0$ along the walls, and it diverges due to the $1 / \sqrt{v_{i, n}}$ factor.

We may also define a diffracted out-state: i.e., the wave function in the junction which is connected through the Kirchhoff formula with the outgoing lead wave function $\psi_{j, m}^{\text {lead,out }}$. This diffracted out-state $\psi_{j, m}^{\text {out,diff }}(\mathbf{q})$ is a circular wave approaching the center of the $j$ th lead with an appropriate angular modulation. It is the complex conjugate of $\psi_{j, m}^{\text {in,diff }}$,

$$
\psi_{j, m}^{\text {out,diff }}=\frac{e^{-i k_{F} r_{j}}}{\sqrt{r_{j}}} \Theta_{j, m}^{\text {out }}\left(\theta_{j}\right)=\frac{e^{-i k_{F} r_{j}}}{\sqrt{r_{j}}}\left(\Theta_{j, m}^{\text {in }}\left(\theta_{j}\right)\right)^{*} .
$$

\section{Semiclassical propagation inside the junction}

Inside the junction, the wave function propagates in a manner that is consistent with the semiclassical approximation (the wavelength is short compared to the size of the junction). The semiclassical method for propagating a wave function goes as follows (Figs. 1 and 5). An initial curve corresponding to an initial wave front is defined. In our case the curve is the circle of radius $r_{b}$ at the center of lead $i$. Trajectories are launched perpendicular to this wave front at all angles $-(\pi / 2)<\theta_{i}<\pi / 2$. At any point $\mathbf{q}$ inside the junction, the wave function $\psi(\mathbf{q})$ is a sum of terms $\left\{\psi_{l}(\mathbf{q}), l=1,2, \ldots\right\}$, one for each trajectory $l$ that arrives at the point $\mathbf{q}$ from a corresponding point $\mathbf{q}_{i, l}(\mathbf{q})=\left(r_{i}=r_{b}, \theta_{i}=\theta_{i, l}\right)$ on the initial arc,

$$
\psi(\mathbf{q})=\sum_{l} \psi_{l}(\mathbf{q})
$$

The wave function associated with trajectory $l$ is

$$
\psi_{l}(\mathbf{q})=\psi^{(0)}\left(\mathbf{q}_{i, l}\right) \mathcal{A}_{l}(\mathbf{q}) \exp \left[i S_{l}(\mathbf{q}) / \hbar-i \mu_{l} \pi / 2\right]
$$

Here $\psi^{(0)}\left(\mathbf{q}_{i, l}(\mathbf{q})\right)$ is the wave function at the point $\mathbf{q}_{i, l}(\mathbf{q})$ which comprises the initial conditions of the $l$ th trajectory. In our case this wave function is the Kirchhoff-diffracted instate corresponding to the lead wave function $\psi_{i, n}^{\text {lead,in }}$,

$$
\psi^{(0)}\left(\mathbf{q}_{i, l}\right)=\psi_{i, n}^{\text {in,diff }}\left(r_{b}, \theta_{i, l}\right) .
$$

$S_{l}(\mathbf{q})$ is the classical action on the path from $\mathbf{q}_{i, l}$ to $\mathbf{q}$, and since we have free-particle motion with the magnitude of the momentum constant, this action is equal to the Fermi momentum times the length of the path $L_{l}(\mathbf{q})$

$$
S_{l}(\mathbf{q})=\int_{\mathbf{q}_{i, l}}^{\mathbf{q}} \mathbf{p} \cdot d \mathbf{q}=p_{F} L_{l}(\mathbf{q})
$$

The amplitude $\mathcal{A}(\mathbf{q})$ is related to the classical density of particles at the point q. It can be computed from certain 


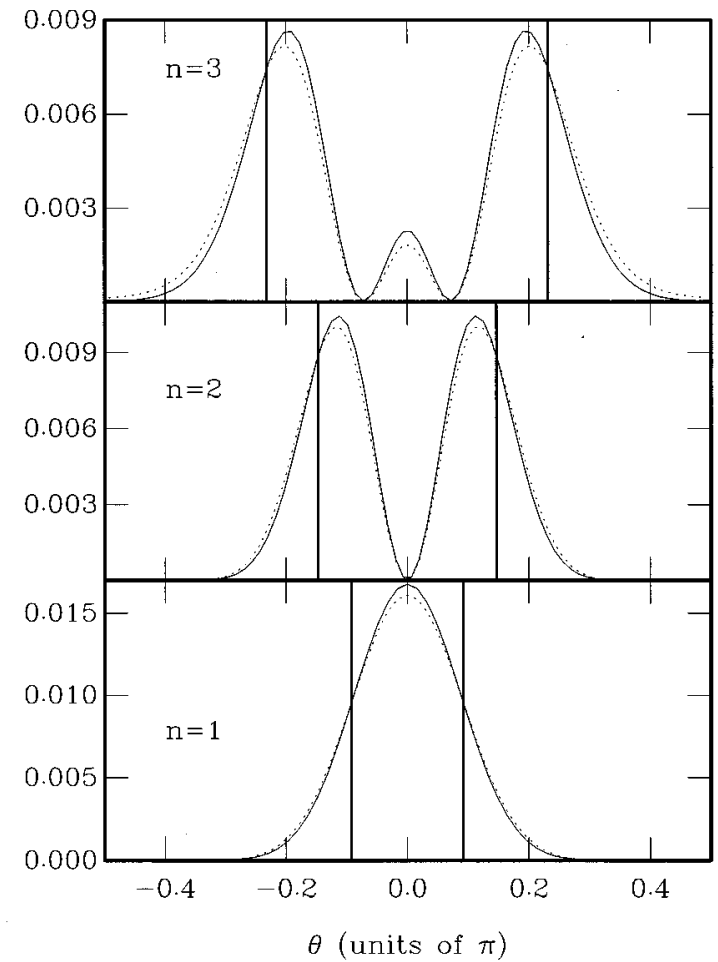

FIG. 4. Angular distribution of the diffracted wave function. $\left|\psi_{i, n}^{\text {in,diff }}\left(\mathrm{r}=8 \mathrm{w}_{\mathrm{i}}, \theta\right)\right|^{2}$ for three values of $n: n=1$ at $k_{F}=3.5 \pi / w_{i}$, $n=2$ at $k_{F}=4.5 \pi / w_{i}$, and $n=3$ at $k_{F}=4.5 \pi / w_{i}$. The dotted curves are from the Kirchhoff approximation used here, Eq. (16). The solid curves are from an exact calculation described in Appendix $C$. The thick vertical bars represent the exit angles of a classical electron.

Jacobians. Let us think about the whole family of trajectories that emanate from the initial arc, and let us consider the instantaneous position on one of these trajectories $\mathbf{q}=(x, y)$ to be a function of time and of the angle $\theta_{i}$ on the initial circle,

$$
\mathbf{q}=\mathbf{q}\left(t, \theta_{i}\right)=\left(x\left(t, \theta_{i}\right), y\left(t, \theta_{i}\right)\right) .
$$

Define

$$
J(\mathbf{q})=J\left(t, \theta_{i}\right)=\frac{\partial}{\partial\left(t, \theta_{i}\right)}\left(x\left(t, \theta_{i}\right), y\left(t, \theta_{i}\right)\right) .
$$

Derivatives such as $\partial x\left(t, \theta_{i}\right) / \partial t$ are components of instantaneous velocity on the path. Derivatives such as $\partial x\left(t, \theta_{i}\right) / \partial \theta_{i}$ can be computed as finite differences $\Delta x\left(t, \theta_{i}\right) / \Delta \theta_{i}$ by integrating two adjacent trajectories to time $t^{28}$

Then the amplitude factor $\mathcal{A}_{l}(\mathbf{q})$ is given by a ratio of Jacobians,

$$
\begin{aligned}
\mathcal{A}_{l}(\mathbf{q}) & =\left|J\left(\mathbf{q}_{i, l}(\mathbf{q})\right) / J(\mathbf{q})\right|^{1 / 2} \\
& =\left|J\left(t=0, \theta_{i}\right) / J\left(t, \theta_{i}\right)\right|^{1 / 2} .
\end{aligned}
$$

Finally, $\mu_{l}$ is the Maslov index for each path, and gives the phase shift associated with caustics or focal points encountered on each path. In the present case, the Maslov index is incremented by two for each bounce off the hard walls, and one for each focus. For the circular junction, there is one

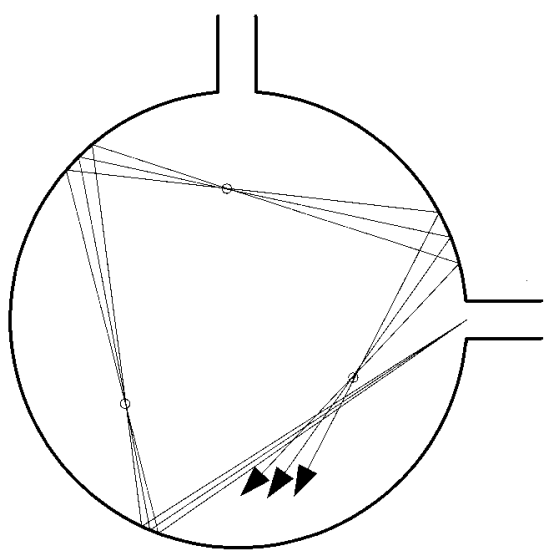

FIG. 5. Three trajectories with similar $\theta_{i}$. There is a focus between each bounce after the first one. The foci are circled in this figure. At each focus, the Maslov index increases by 1 . For each bounce off a hard wall, the Maslov index increases by 2 (if the walls were soft, it would increase by 1 ).

focus between each bounce after the first (Fig. 5 shows this geometrically, and it can be verified by analytic evaluation of the Jacobian).

As the electron continues to bounce around the enclosure, a large number of paths can contribute to the wave function at each point. In the semiclassical method, we cut off this sum over paths in some way, for example by including only paths of length less than some maximum. The hypothesis is that conductance fluctuations are dominated by interference among waves that follow relatively short paths from entrance to exit. This hypothesis is partially supported by our calculations.

\section{E. Wave function near the exit lead}

To calculate the $S$-matrix elements from semiclassical wave functions, we identify paths that go from the center of the entrance lead $i\left(x_{i}=0, y_{i}=0\right)$ to the center of any lead $j\left(x_{j}=0, y_{j}=0\right)$, including the entrance lead itself, $j=i$. There is a discrete set of such paths, and each is labeled by the index $l$. The wave function associated with each path at the final point is given by combining Eqs. (21)-(26) with Eq. (16),

$$
\begin{aligned}
\psi_{l}\left(x_{j}\right. & \left.=0, y_{j}=0\right) \\
& =\frac{e^{i\left(k_{F} L_{l}-\mu_{l} \pi / 2\right)}}{\sqrt{r_{b}}} \mathcal{A}_{l}\left(x_{j}=0, \quad y_{j}=0\right) \Theta_{i, n}^{\text {in }}\left(\theta_{i, l}\right) .
\end{aligned}
$$

Here $k_{F} L_{l}$ is the phase associated with the entire length of the $l$ th path from the center of the entrance to the center of the exit. It arises by combining $\exp \left(i k_{F} r_{i}\right)$ in Eq. (16) with the action integral term (23) which goes from the initial circle to the final point.

We make the approximation that near this final point, but, inside the junction, the wave function $\psi_{l}\left(x_{j}, y_{j}\right)$ is a plane wave approaching the lead from angle $\theta_{j, l}$ :

$$
\psi_{l}\left(x_{j}>0, y_{j}\right)=\psi_{l}\left(x_{j}=0, y_{j}=0\right) \exp \left[i \mathbf{p}_{j, l} \cdot \mathbf{q}_{j} / \hbar\right],
$$


where $\mathbf{p}_{j, l}$ is the momentum at lead $j$ on the path $l$. The rays associated with this plane wave are all parallel to this final momentum. With this approximation, let us evaluate the Jacobians in Eq. (26). On the initial arc,

$$
J\left(t=0, \theta_{i}\right)=\left|\frac{\partial(x, y)}{\partial\left(t, \theta_{i}\right)}\right|=\left|r \frac{\partial\left(r, \theta_{i}\right)}{\partial\left(t, \theta_{i}\right)}\right|=r_{b} v_{F} .
$$

At the final point,

$$
\begin{aligned}
J\left(t_{f}, \theta_{i}\right) & =\left|\frac{\partial(x, y)}{\partial\left(t, \theta_{i}\right)}\right|=\left|\frac{\partial\left(x_{j}, y_{j}\right)}{\partial\left(t, \theta_{i}\right)}\right| \\
& =\left|\frac{\partial x_{j}}{\partial t} \frac{\partial y_{j}}{\partial \theta_{i}}-\frac{\partial y_{j}}{\partial t} \frac{\partial x_{j}}{\partial \theta_{i}}\right| \\
& =v_{F}\left|\cos \theta_{j, l}\left(\frac{\partial y_{j}}{\partial \theta_{i}}\right)_{t}-\sin \theta_{j, l}\left(\frac{\partial x_{j}}{\partial \theta_{i}}\right)_{t}\right| \\
& =v_{F} \cos \theta_{j, l}\left|\left(\frac{\partial y_{j}}{\partial \theta_{i}}\right)_{x}\right| .
\end{aligned}
$$

The last of these formulas follows from

$$
\begin{aligned}
\left.\frac{\partial y_{j}}{\partial \theta_{i}}\right|_{t} & =\left.\frac{\partial y_{j}}{\partial \theta_{i}}\right|_{x}+\left.\frac{\partial y_{j}}{\partial x_{j}}\right|_{\theta_{i}} \frac{\partial x_{j}}{\partial \theta_{i}} t=\left.\frac{\partial y_{j}}{\partial \theta_{i}}\right|_{x}+\left.\frac{\partial y_{j} /\left.\partial t\right|_{\theta_{i}}}{\partial x_{j} /\left.\partial t\right|_{\theta_{i}}} \frac{\partial x_{j}}{\partial \theta_{i}}\right|_{t} \\
& =\left.\frac{\partial y_{j}}{\partial \theta_{i}}\right|_{x}+\left.\tan \theta_{j, l} \frac{\partial x_{j}}{\partial \theta_{i}}\right|_{t} .
\end{aligned}
$$

Equation (30) has the following meaning. Let us define a coordinate $s$ that goes around the circumference of the junction, passing straight across the leads. From the initial circle, we find the $l$ th path that lands at the center of the $j$ th lead. Then we increment $\theta_{i}$ and ask where on the boundary the adjacent path lands. The derivative $\left|\partial y_{j} / \partial \theta_{i}\right|_{x}$ is equivalent to $\left|\partial s / \partial \theta_{i}\right|$ for that path. Then the amplitude factor for the wave function is

$$
\frac{1}{\sqrt{r_{b}}} \mathcal{A}_{l}\left(x_{j}=0, y_{j}=0\right)=\left|\cos \theta_{j, l} \frac{d s_{l}}{d \theta_{i}}\right|^{-1 / 2} .
$$

Combining Eqs. (27), (28), and (30), the wave function near the $j$ th lead is a sum of plane waves

$$
\psi_{l}\left(\mathbf{q}_{j}\right)=\exp \left(i \mathbf{p}_{j, l} \cdot \mathbf{q} / \hbar\right) \mathcal{T}_{l} \Theta_{i, n}^{\text {in }}\left(\theta_{i, l}\right),
$$

where

$$
\mathcal{T}_{l}=\left|\cos \theta_{j, l} \frac{d s_{l}}{d \theta_{i}}\right|^{-1 / 2} \exp \left(i k_{F} L_{l}-i \mu_{l} \pi / 2\right) .
$$

\section{F. Semiclassical formula for the $S$ matrix}

We use this semiclassical approximation [Eq. (33)] for the wave function to represent $\psi_{i, n}^{+}$in Eq. (12) near the exit lead. Now we use Eq. (12) to calculate the $S$-matrix element. As stated in Sec. III B, this $S$-matrix element is calculated by integration around a half-circle centered on the $j$ th lead. The integral is evaluated using the stationary-phase approximation.
On the arc around the mouth of lead $j, r_{j}=r_{b}$, the state $\psi_{j, m}^{\text {out }}$ is approximately equal to the Kirchhoff wave function $\psi_{j, m}^{\text {out,diff }}$ defined in Eq. (19). When we apply the stationaryphase approximation we examine the $\theta_{j}$ dependence of the phase of the plane-wave contribution of each trajectory,

$$
\Phi_{l}\left(\theta_{j}\right) \equiv \mathbf{p}_{j, l} \cdot \mathbf{q} / \hbar=-k_{F} r_{b} \cos \left(\theta_{j}-\theta_{j, l}\right) .
$$

This phase is stationary when $\theta_{j}$, the angle defining points on the arc, is equal to $\theta_{j, l}$, the direction from which the $l$ th trajectory approaches the lead. The second derivative of the phase at this point is

$$
\frac{d^{2} \Phi_{l}}{d \theta_{j}^{2}}=k_{F} r_{b}>0
$$

and it follows that

$$
\int d \theta_{j} \exp \left(i \mathbf{p}_{j, l} \cdot \mathbf{q} / \hbar\right) \approx e^{-i k_{F} r_{b}}\left(\frac{2 \pi}{k_{F} r_{b}}\right)^{1 / 2} e^{i \pi / 4} .
$$

At the stationary phase point, $\partial \psi / \partial r_{j}=-i k_{F} \psi$, both for $\psi=\psi_{l}$ and for $\psi=\psi_{j, m}^{\text {out,diff }}$. Everything else in the integral in Eq. (12) is taken to be constant.

Now using Eqs. (33) and (19) in Eq. (12), and applying Eq. (37), the result is that the contribution to an $S$-matrix element from the $l$ th trajectory is

$$
\sqrt{2 \pi k_{F}} \frac{\hbar}{M} e^{i \pi / 4} \Theta_{i, n}^{\text {in }}\left(\theta_{i, l}\right) \mathcal{T}_{l} \Theta_{j, m}^{\text {in }}\left(\theta_{j, l}\right)
$$

or, in full and in abbreviated notation,

$$
\begin{aligned}
S_{m n}^{(j i)}= & m n \sqrt{2 w_{i} w_{j}} \pi^{3 / 2} e^{i 3 \pi / 4} \frac{k_{F}^{3 / 2}}{\sqrt{k_{i, n} k_{j, m}}} \sum_{l}\left[\cos \left(\theta_{i, l}\right)+\frac{k_{i, n}}{k_{F}}\right] \\
& \times\left[\cos \left(\theta_{j, l}\right)+\frac{k_{j, m}}{k_{F}}\right] \chi_{n}\left(\frac{w_{i} k_{F}}{2} \sin \theta_{i, l}\right) \chi_{m}\left(\frac{w_{j} k_{F}}{2} \sin \theta_{j, l}\right) \\
& \times \frac{1}{\sqrt{\cos \theta_{j, l}}}\left|\frac{\partial s_{l}}{\partial \theta_{i}}\right|^{-1 / 2} \exp \left(\frac{i}{\hbar} \int_{\mathbf{q}_{l}} \mathbf{p} \cdot d \mathbf{q}-i \frac{\pi}{2} \mu_{l}\right) \\
= & \sum_{l} a_{l} \exp \left(i k_{F} L_{l}\right)
\end{aligned}
$$

The same result for the $S$ matrix can be obtained by another method. Instead of evaluating $\psi_{i, n}^{+}$and $\psi_{j, n}^{\text {out,diff }}$ on an arc, we could continue the plane-wave representation of $\psi_{i, n}^{+}$up to the lead mouth, and combine it with $\psi_{j, m}^{\text {lead,out }}$ in Eq. (12), integrating straight across the lead mouth instead of along the arc. These two approaches are consistent with the Kirchhoff approximation. ${ }^{29}$

The power spectrum of $S_{m n}^{(j i)}\left(k_{F}\right)$ is defined as

$$
\hat{S}_{m n}^{(j i)}(L) \equiv\left|\int_{0}^{k_{F}^{\max }} e^{-i k_{F} L} S_{m n}^{(j i)}\left(k_{F}\right) d k_{F}\right|^{2} .
$$

It follows from Eq. (40) that $\hat{S}_{m n}^{(j i)}(L)$ is a set of peaks at $L_{l}$ (the length of the $l$ th trajectory from the center of the entrance to the center of the exit). The height of each peak should be proportional to the absolute square of the preexponential factor $a_{l}$ in Eq. (40). 


\section{G. Comparison with previous semiclassical theory}

Baranger, Jalabert, and Stone, ${ }^{11}$ along with Lin and Jensen, ${ }^{16}$ developed a similar (BJSLJ) semiclassical theory in which the initial quantum state was taken as the undiffracted lead state. In our theory, the initial wave front is circular as a result of diffraction, and trajectories are launched from the center of the lead mouth in all directions. In the BJSLJ theory, the initial wave front is a straight line, and the classical trajectories are launched in only the two classical directions dictated by the parallel and transverse momenta of the particular mode in the lead.

For leads with large lead widths compared to the characteristic dimensions of the junction (strongly open junctions), and for wavelengths short in comparison with these lengths, we expect that the BJSLJ formulas are appropriate. Whenever diffraction is important (whenever the wavelength is comparable to the size of the leads), the present theory should be better. On the other hand, some of our formulas assume that the lead is narrow compared to the size of the junction; this assumption is not made in BJSLJ.

A nice feature of our theory is that the contribution to the $S$ matrix from each path is given by the same formula, Eq. (39). The BJSLJ theory requires two different expressions depending on whether the path has encountered a curved wall between the entrance and exit.

\section{COMPARISON WITH QUANTUM RESULTS}

Ishio and Burgdörfer ${ }^{13}$ carried out a fully quantummechanical calculation of the conductance of a circular junction. They showed that the Fourier transform of the transmission amplitude displays distinct peaks at frequencies corresponding to trajectories connecting the entrance and exit leads.

We originally intended to compare our semiclassical formulas with their quantum results. However, we found that their method (even with the improvements incorporated in Ref. 6) did not give results sufficiently precise and reliable to allow a definitive comparison of peak heights. Therefore, we developed an improvement upon their method, using an expansion in appropriately normalized Bessel functions rather than plane waves. This method is described in Appendix A. Below we show results of our calculations and comparisons with semiclassical formulas.

Following Ref. 13 the system consists of a circular junction of radius $R=\sqrt{1+4 / \pi}$, joined at a right angle to two wire leads of width $w=0.0935 \sqrt{\pi+4}$. For purposes of calculating the classical trajectories, the lead mouths were taken as curved surfaces, continuations of the circle. This circle geometry is particularly convenient because all of the properties of trajectories can be calculated in closed form. We compared our semiclassical theory with exact quantum results over the energy range corresponding to $w k_{F} / \pi=[1 \ldots 5]$ with zero magnetic field; in this range the radius to wavelength ratio is $R / \lambda=[3 \ldots 15]$.

If we start a large number of trajectories on the initial arc around the entrance lead mouth, and allow them to bounce around the junction 15 times, we find that about $70 \%$ of the trajectories have hit the mouth of one of the leads and therefore have left the junction. Our semiclassical calculation includes only such trajectories, so it necessarily gives transmis-

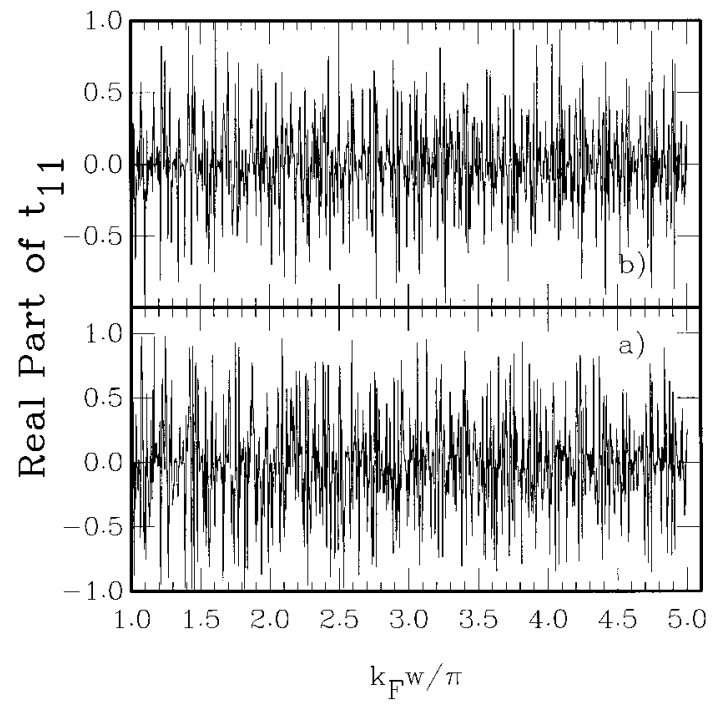

FIG. 6. Real part of the transmission amplitude. (a) Quantum. (b) Semiclassical. The transmission amplitude fluctuates wildly as a function of $k_{F}$, and little correspondence between quantum and semiclassical calculations is visible.

sion and reflection coefficients that are too small.

Accordingly, the purpose of the semiclassical calculation is not to reproduce $S_{m n}^{(j i)}$ but rather to reproduce its largescale structure. From Eq. (39) we see that fluctuations of $S_{m n}^{(j i)}\left(k_{F}\right)$ having long wavelength on the $k_{F}$ axis arise from relatively short trajectories. To see this structure, it is best to take a Fourier transform.

\section{A. Transmission coefficient $t_{11}$}

Figure 6 shows the real part of the semiclassical and quantum transmission amplitude $t_{11} \equiv S_{11}^{(21)}$ as a function of $k_{F}$. This quantity is the amplitude for transmission from the lowest mode of lead 1 to the lowest mode of lead 2. The semiclassical version employs 120 trajectories (up to 15 bounces). Both the quantum and semiclassical results are highly oscillatory, and it is hard to see any correspondence between them.

Examination of the two curves shows three discrepancies. (1) The amplitude of the semiclassical curve is generally too small. This is due to the finite number of trajectories used in this calculation. (2) The semiclassical formula diverges as small $k_{F}$ (near the threshold where $k_{F} w / \pi=1$ ) due to a breakdown of the Kirchhoff approximation for diffraction at the lead mouths [Eq. (16) contains a factor of $\sqrt{v_{i, n}}$ in the denominator; this factor goes to zero at threshold]. This breakdown could be eliminated if a better expression for the diffracted wave function were obtained, but the failure occurs in only a very small range of $k_{F}$, and it is not visible in Fig. 6. (3) The magnitude of the semiclassical $t_{11}$ is not necessarily less than 1: semiclassical formulas are not necessarily unitary.

Despite all of this, the large-scale structure of the fluctuations of $t_{11}$ is correctly described by the semiclassical formula. This is best shown by examination of the Fourier transform.

The power spectrum of the $t_{11}\left(k_{F}\right)$ in Fig. 6 is denoted $\hat{t}_{11}$, and is shown in Fig. 7. The agreement between quantum 


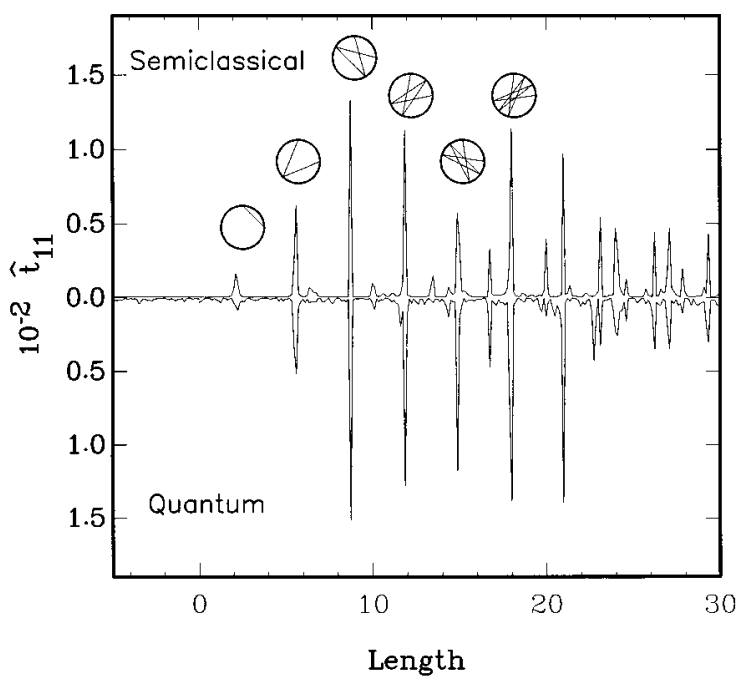

FIG. 7. Power spectrum of the transmission amplitude. Each major peak corresponds to one of the trajectories between entrance and exit.

and semiclassical results up to $L \sim 21$ is remarkable, and, above $L \sim 21$, there is still substantial agreement. Each major peak corresponds to an identifiable path from the center of the entrance to the center of the exit. The location of the peak is the length of the path, and the height of the peak is given accurately by our formula Eq. (39).

As we go to longer lengths some discrepancies arise. We believe that this is primarily due to the fact that we use a finite number of trajectories in the semiclassical calculation. [There is also some possibility that numerical errors contribute to the high-frequency (long-length) fluctuations in the quantum calculations.]

As reported in Ref. 13, we see that the "asterisk" trajectories dominate the power spectrum. Some of these are shown as insets in Fig. 7 at the corresponding peak positions. "Asterisks" are those trajectories which, for a given number of bounces between entrance and exit, have the smallest angles (those angles closest to the lead normals) at the entrance and exit leads. The reason for the importance of these trajectories lies in the entrance geometry and the mode number. The entering electrons are rather well collimated in the forward direction, and the amplitude of the diffracted wave function falls off rapidly with increasing angle. Furthermore, for mode $n=1$, the diffracted wave function has no nodes in this direction.

"Whispering gallery" trajectories, which run along the edges of the walls, do not play an important role here. For example, the shortest path from entrance to exit has an initial angle at $45^{\circ}$ from the normal; this produces the small peak in Fig. 7 at $L \sim 2$. There is an infinite family of similar paths which travel one-fourth of the way around the circle; each successive member has a larger initial angle (between $45^{\circ}$ and $90^{\circ}$ ) and one more bounce against the wall. The limiting length of these trajectories is $\frac{1}{4} 2 \pi R(\sim 2.4)$, but there is no peak there in Fig. 7. Again, this happens because electrons leaving the lead are collimated in the forward direction by the entrance geometry.

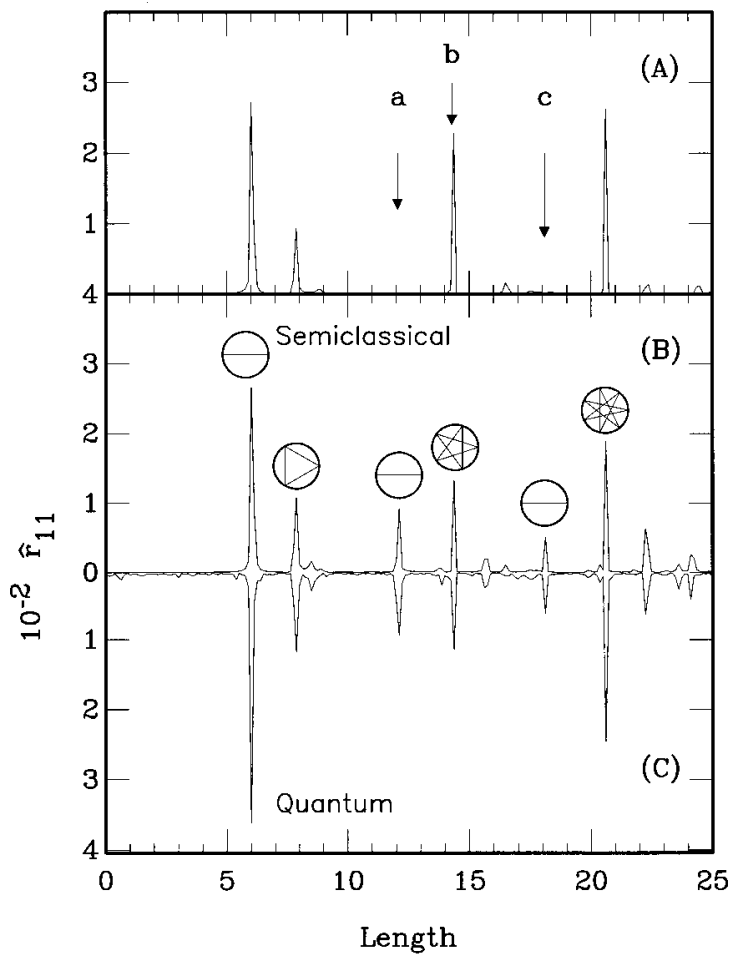

FIG. 8. Power spectra of the semiclassical and quantum reflection amplitudes. (A) Semiclassical without diffractive scattering from lead mouths. (B) Semiclassical including diffractive scattering. (C) Quantum. Again, the major peaks arise from identifiable paths. Peaks $a$ and $c$ are missing in the simple semiclassical calculation in panel (A). They are "'ghost paths,' caused by diffractive reflection from the mouth of a lead. Waves go from the entrance lead across the junction, and are reflected back to the entrance lead; there they are partially absorbed and partially reflected with diffraction. Peak $a$ represents four traverses, and peak $c$ six traverses of the diameter of the circle.

\section{B. Reflection coefficient $r_{11}$ : diffractive scattering and "ghost paths"}

The power spectrum of the reflection coefficient $\left(r_{11} \equiv S_{11}^{(11)}\right)$ is shown in Fig. 8. Again, the locations and heights of several of the major peaks are accurately predicted by the semiclassical formula [Fig. 8(A)]. For example, the lowest-frequency peak arises from the "straight-ahead" path, which bounces once from the far wall before going directly back out the entrance, and has a length of $4 R$.

On the other hand, certain interesting discrepancies appear between Figs. 8(A) and 8(C). Peaks marked $a$ and $c$ are not present in the semiclassical calculation, while peak $b$ has the wrong magnitude.

Peak $a$ occurs at a length exactly twice that of the straight-ahead path $(8 R)$. We may guess, then, that this peak arises from waves which propagate across the circle and back, but then they are not totally absorbed by the entrance aperture. These waves are partially absorbed, but also partially reflected diffractively from the aperture and they retrace the straight-ahead path.

In the simple semiclassical calculations [Figs. 7 and 8(A)], when a trajectory reaches a lead mouth it is assumed that all of the flux associated with that trajectory is absorbed: 


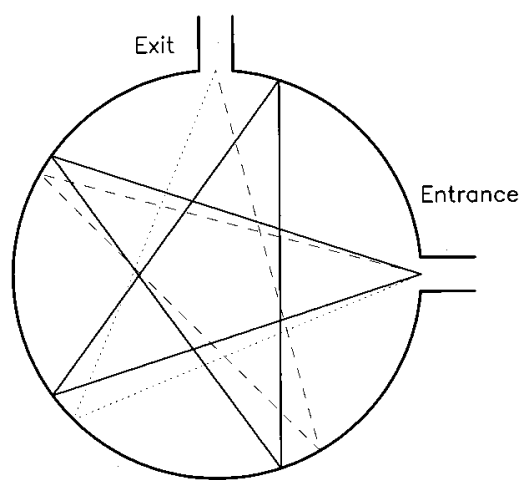

FIG. 9. Three trajectories which contribute to peak $b$ of Fig. 8 . The heavy line is a star-shaped trajectory that bounces specularly around the enclosure, and produces peak $b$ in Fig. 8(A). The dashed lines forming the distorted star together constitute a pair of "ghost paths." One path has one bounce from entrance to exit, diffractive reflection from the exit, and then two bounces back to the entrance. The other member of the pair is the reverse of this path. The distorted star destructively interferes with the star, reducing the height of peak $b$ in Fig. 8(B).

the lead casts a sharp shadow in the reflected wave. In reality, diffractive scattering blurs these shadows. A diffracted wave goes out in all directions; it only partially cancels the specularly reflected wave, and it produces nonzero amplitude on paths that are not permitted by geometrical optics.

Therefore, diffractive scattering produces peaks associated with "ghost paths" that bounce elastically around the enclosure and diffract one or more times off a lead mouth. Diffractive reflection from an opening can be treated by Babinet's principle: the total reflected wave is the specularly reflected wave minus the wave produced by a small barrier coinciding with the opening.

In Appendix B we give an approximate formula for this diffractive scattering. With this formula we have recalculated $t_{11}\left(k_{F}\right)$ and $r_{11}\left(k_{F}\right)$. These calculations include trajectories of up to 15 bounces which travel from entrance to exit, entrance to entrance, and exit to exit. The trajectories are connected by up to two scattering events as specified in Appendix B. In addition, we discard those trajectories whose cumulative length is longer than 50: this is the Nyquist frequency from the grid of $k_{F}$ used in our quantum calculations.

The results of a calculation including diffractive scattering are shown in Fig. 8(B). Now there is excellent agreement between the quantum and semiclassical calculations. Peaks $a$ and $c$, respectively, represent four and six traverses of the circle.

Peak $b$ arises from an interesting effect. The largest contribution to this peak arises from the star-shaped five-bounce trajectory from entrance to entrance, with length $\sim 14.3$ (heavy line in Fig. 9). However, a significant contribution also comes from two trajectories which go from entrance to exit, where they are connected by diffractive scattering. These trajectories are shown by the dashed and dotted curves in Fig. 9, and have a total length approximately equal to that of the star trajectory. They interfere destructively with the star-shaped trajectory and reduce the magnitude of peak $b$. In effect, a portion of the wave front associated with the starshaped trajectory is absorbed by the exit lead, and the dif-

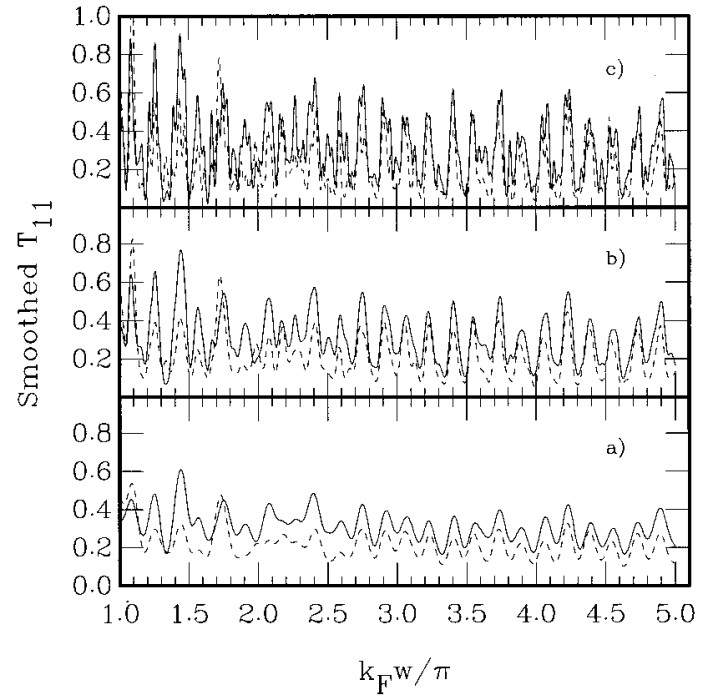

FIG. 10. Gaussian smoothed quantum (solid) and semiclassical (dotted) transmission probability. (a) $\sigma=\pi / 5$, (b) $\sigma=\pi / 10$, (c) $\sigma=\pi / 20$.

fracted "shadow" of the lead mouth reduces the amplitude of the wave function reflected out the entrance lead.

\section{Squares of matrix elements, $\left|S_{11}^{(j i)}\right|^{2}$}

A far more severe test of the semiclassical formula comes from examination of the absolute squares of the $S$-matrix elements, $S_{m n}^{(j i)}$, the transmission and reflection probabilities. These are directly related to the measurable conductance by Eq. (1).

The large-scale structure of $S_{m n}^{(j i)}\left(k_{F}\right)$ arises from short paths, but the same formula, Eq. (40), predicts that the largescale structure of $\left|S_{m n}^{(j i)}\left(k_{F}\right)\right|^{2}$ comes from pairs of paths with short length differences:

$$
\left|S_{m n}^{(j i)}\left(k_{F}\right)\right|^{2}=\sum_{l_{1}, l_{2}} a_{l_{1}}^{*} a_{l_{2}} e^{i k_{F}\left(L_{l_{2}}-L_{l_{1}}\right)} .
$$

Pairs of very long paths can have short length differences, so any scheme in which long paths are omitted might fail completely. In fact, we will see that short path semiclassical calculations are partially successful.

Quantum and semiclassical Gaussian-smoothed plots of the transmission probability $T_{11}=\left|t_{11}\right|^{2}$ are shown in Fig. 10 for Gaussian widths $\sigma$ corresponding to cutoff frequencies (lengths) of 5, 10, and 20,

$$
\text { Smoothed } T_{11}=\int d k_{F}^{\prime} T_{11}\left(k_{F}^{\prime}\right) e^{-\left(k_{F}-k_{F}^{\prime}\right)^{2} / \sigma^{2}} .
$$

One again notes that the semiclassical result tends to have a slightly smaller amplitude than the quantum version. Nevertheless, the quantum and semiclassical results match rather well in phase and amplitude: the large-scale structure of $T_{11}\left(k_{F}\right)$ is rather well predicted by our short-path semiclassical calculation ( $\leqslant 15$ bounces, total length $<50$, two diffractive scattering events).

Figure 11(A) shows $\hat{T}_{11}$, the power spectrum of both the quantum and the semiclassical transmission probability. ${ }^{30}$ It 

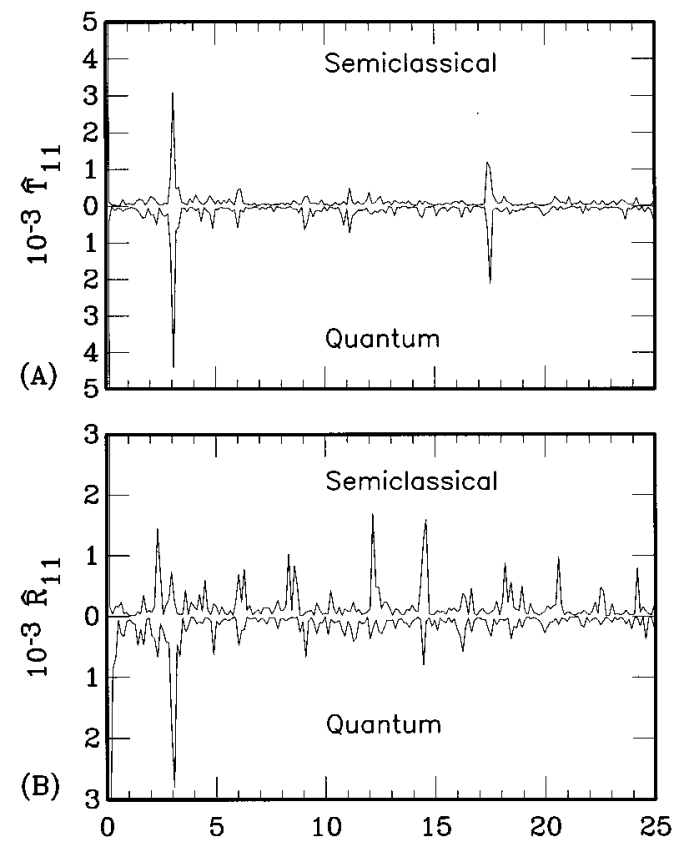

FIG. 11. Power spectra of (A) the transmission probability, and (B) the reflection probability.

corroborates the good agreement gleaned from inspection of the smoothed plots in Fig. 10. One surprising aspect of these results is the lack of structure present in $T_{11}$ relative to that in $t_{11}$.

There are very many pairs of paths with short length differences, and they contribute a very large number of peaks. Many of these peaks interfere destructively, however, so there remain only two sizable (non-dc) peaks in Fig. 11(A).

The largest peak in Fig. 11(A), at $L \sim 3$, corresponds to the length difference of successive asterisk trajectories having a large number of bounces. That length difference converges to the diameter of the circle. The peak at $\sim 17.5$ is due in part to interference between entrance-to-exit asterisk trajectories (recall that these are the longest trajectories for a given number of bounces) with $n$ bounces and the secondlongest trajectories with $n+6$ bounces.

Overall, the agreement between semiclassical and quantum results in Fig. 11(A) is good enough that the semiclassical formula is credible for this case. However, when we examine the square of the reflection coefficient $R_{11}=\left|r_{11}\left(k_{F}\right)\right|^{2}$, the results are not very good [Fig. 11(B)]. Both quantum and semiclassical spectra show more structure than is present in $T_{11}$, but the peaks differ significantly in position and magnitude. This lack of agreement should be contrasted with the nice agreement for the power spectrum of $r_{11}$ in Fig. 8(B).

Why does the semiclassical formula give a good approximation to the power spectra of transmission and reflection amplitudes (Figs. 7 and 8) and a decent approximation to the power spectrum of the transmission probability [Figs. 10 and 11(A)], but a poor representation of the reflection probability? Two possible explanations are available. (1) Diffractive scattering is more important for reflection than for transmission, and our approximate formulas for this process are not yet sufficiently accurate. (2) For some reason, long paths are more important for reflection than for transmission, and trun- cation of the sum over classical paths causes the discrepancy. We have studied these issues at some length, and have not yet reached a conclusion. In the Sec. V, we offer a hypothesis which, if correct, will make this problem less relevant to experimental comparisons.

\section{A HYPOTHESIS ABOUT LONG PATHS}

Even in a semiconductor microstructure with no defects or impurities, thermal fluctuations disturb the electrons as they bounce around, distorting the paths and causing a loss of coherence. We expect that long paths through the junction may be more disturbed by thermal fluctuations than short paths. It is credible, then, to suggest that in experimental measurements, interference fluctuations associated with short length differences between long paths may be quenched by thermal fluctuations. If this hypothesis is correct, then for comparison with measurements, Eq. (42) should be replaced by

$$
\left|S_{m n}^{(j i)}\left(k_{F}\right)\right|^{2}=\left|S_{m n}^{(j i)}\left(k_{F}\right)\right|_{\mathrm{inc}}^{2}+\sum_{l_{1}, l_{2}} a_{l_{1}}^{*} a_{l_{2}} e^{i k_{F}\left(L_{l_{2}}-L_{l_{1}}\right)} f_{l} f_{l^{\prime}},
$$

where $\left|S_{m n}^{(j i)}\left(k_{F}\right)\right|_{\text {inc }}^{2}$ is an incoherent "background" contribution, and $f_{l}$ is a function which cuts off long paths, such as $\exp \left[-(L / \Lambda)^{2}\right]$. The hypothesis is that because of thermal fluctuations, a reduced semiclassical sum like Eq. (44) could be a better representation of measured interference fluctuations than the full semiclassical sum Eq. (42), which in principle includes small path differences between arbitrarily long paths.

How do we express this same hypothesis in terms of the quantum $S$ matrix? If there are no thermal fluctuations, we would simply compute the power spectrum of $T_{m n}\left(k_{F}\right)$ as in Fig. 11(A). Naively, we might think that thermal fluctuations would act to smooth $\left|S_{m n}^{(j i)}\left(k_{F}\right)\right|^{2}$, and thereby simply reduce or remove its high-frequency (large $\Delta L$ ) components.

However, the hypothesis is that thermal fluctuations reduce coherence in long paths more than in short paths. This hypothesis implies that we should first smooth $S_{m n}^{(j i)}$ eliminating its high-frequency components, and then square the smooth result to obtain a partially coherent $\left|S_{m n}^{(j i)}\left(k_{F}\right)\right|^{2}$. We propose that "smoothing then squaring" may give a better representation of experimentally measured interference fluctuations than "squaring then smoothing." Such calculations are shown in Figs. 12(A) and 12(B) (related procedures were suggested in Refs. 24 and 13).

In this case, this procedure improves the agreement between semiclassical and quantum calculations. The semiclassical formula for the power spectrum of the transmission probability agrees reasonably well with the quantum result, and the semiclassical formula for the reflection probability [Fig. 12(B)] is much improved over that seen in Fig. 11(B). However, this improved agreement by itself does not justify the hypothesis. Experimental tests of this hypothesis could occur when conductance fluctuations dependent only upon geometry are obtained. In any case, the proposed semiclassical formula accurately predicts the large-scale structure of $S$-matrix elements. 

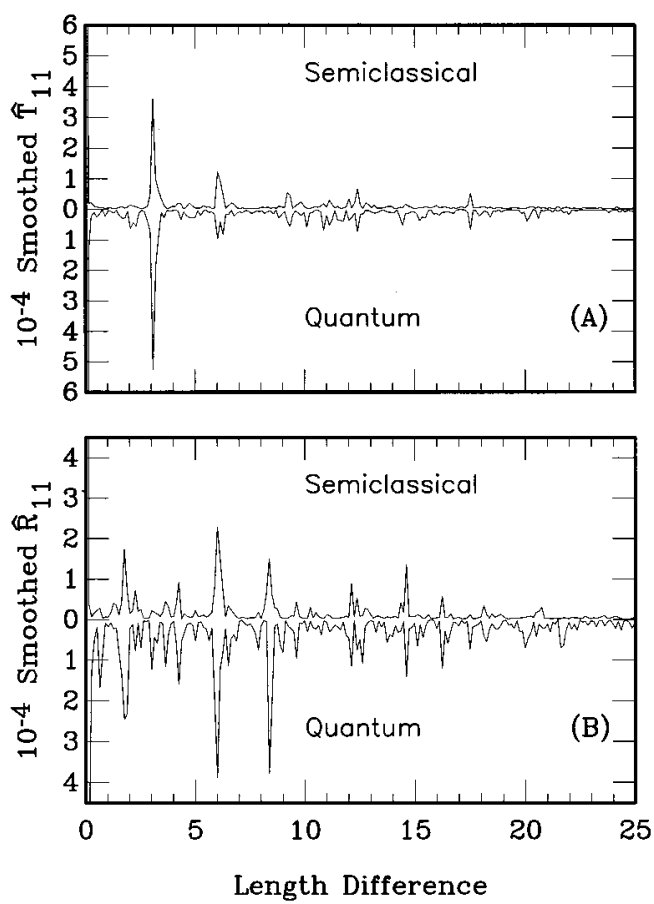

FIG. 12. Quantum-mechanical and semiclassical power spectra of (A) the transmission probability and (B) the reflection probability resulting from first smoothing $S_{\text {II }}^{(i j)}\left(k_{F}\right)$ using a cutoff width of $\sigma=\pi / 40$.

(It is important to draw a distinction between thermal fluctuations in the electron reservoirs or the leads outside the junction, and the thermal fluctuations within the junction itself. Thermal fluctuations from sources outside the junction typically will not reduce coherence on long paths relative to short paths; only thermal fluctuations within the junction itself could have the effect of retaining short-path coherence but reducing long-path coherence.)

\section{SUMMARY}

(1) We have improved upon previous methods for calculating the quantum $S$ matrix for a two-dimensional junction.

(2) We have derived a semiclassical formula for the $S$ matrix of such a junction.

(3) The semiclassical formula gives a good representation of the large-scale structure of the transmission amplitude.

(4) For the reflection amplitude, the semiclassical formula must be modified to include diffractive scattering and "ghost paths." With that modification, it gives a good representation of the large-scale structure of the reflection amplitude.

(5) The semiclassical formula gives a good representation of the large-scale structure of the transmission probability.

(6) At present, this formula gives a poor representation of the quantum reflection probability.

(7) We have offered a hypothesis that thermal fluctuations within the junction may reduce coherence on long paths. If this is correct, then we expect that formulas including only short paths will give a good representation of measured conductance fluctuations.

\section{APPENDIX A: SOLVING FOR THE FULLY QUANTUM $S$ MATRIX}

We used two different methods for the quantum calculations reported in this paper. To calculate the $S$ matrix for the circular junction, we used a basis set expansion described in this appendix. To check the validity of the Kirchhoff approximation, and that of our formula for diffractive scattering (Appendix B), we used the boundary element method described in Appendix C.

For the basis set expansion method we followed the method of Nakamura and Ishio $^{31}$. A similar approach was also employed in Ref. 32. We found that we needed a modification of these previous approaches in order to obtain numerically converged results.

We wish to match a representation of the wave function inside the junction with a different representation which is appropriate outside of the junction. Outside the junction, the wave function is zero except in the leads. The wave function in lead $j$ can be expanded in lead wave functions,

$$
\psi_{\text {lead }}^{(j)}\left(\mathbf{q}_{j} ; n\right)=\delta_{j i} \psi_{i, n}^{\text {lead,in }}\left(\mathbf{q}_{j}\right)+\sum_{m=1}^{M} S_{m n}^{(j i)} \psi_{j, m}^{\text {lead,out }}\left(\mathbf{q}_{j}\right) .
$$

This equation is similar to Eq. (6), but here the sum includes closed channels (evanescent modes) so Eq. (A1) holds everywhere in lead $j$.

The wave function inside the junction is expanded in basis functions $\Phi_{b}(\mathbf{q})$, which are selected solutions to the Schrödinger equation,

$$
\begin{gathered}
\psi_{\text {junc }}(\mathbf{q})=\sum_{b} c_{b} \Phi_{b}(\mathbf{q}), \\
\left(\nabla^{2}+k_{F}^{2}\right) \Phi_{b}(\mathbf{q})=0 .
\end{gathered}
$$

The coefficients $S_{m n}^{(j i)}$ and $c_{b}$ are chosen so that the "inside" and "outside" representations match along the perimeter of the junction. The normal derivatives of each representation must also match along this boundary. Thus

$$
\psi_{\text {junc }}=0
$$

along the walls, and

$$
\begin{gathered}
\psi_{\text {junc }}=\psi_{\text {lead }}^{(j)}, \\
\frac{\partial}{\partial x_{j}} \psi_{\text {junc }}=\frac{\partial}{\partial x_{j}} \psi_{\text {lead }}^{(j)}
\end{gathered}
$$

at the mouth of lead $j$.

Equation (A4c) allows us to express the $S_{m n}^{(j i)}$ in terms of the $c_{b}$. Multiplying both sides by $\phi_{j, m}$ and integrating over the mouth of lead $j\left(x_{j}=0\right)$, results in the expression

$$
S_{m n}^{(j i)}=\delta_{i, j} \delta_{n, m}-\sum_{b} c_{b} \mathbb{F}_{j m b},
$$

where

$$
\mathbb{F}_{j m b}=i \frac{\sqrt{v_{m}}}{k_{m}} \int_{-w_{j} / 2}^{w_{j} / 2} d y_{j} \phi_{j, m}\left(y_{j}\right) \frac{\partial}{\partial x_{j}} \Phi_{b}(\mathbf{q})
$$


Now let us define a coordinate $s, 0 \leqslant s<\Lambda$, that goes around the perimeter of the junction, and straight across the lead mouths. We expand the inside and outside representations in a Fourier series in $s$ with coefficients $\widetilde{\psi}_{\text {junc, } l}$ and $\widetilde{\psi}_{\text {lead }, l}$, respectively (Ref. 31 ). We find

$$
\begin{aligned}
\widetilde{\psi}_{\text {junc }, l} & \equiv \frac{1}{\Lambda} \int_{0}^{\Lambda} d s \psi_{\text {junc }}(s) e^{-i 2 \pi l s / \Lambda} \\
& =\sum_{b} c_{b} \mathrm{G}_{b l},
\end{aligned}
$$

where

$$
\mathrm{G}_{b l}=\frac{1}{\Lambda} \int_{0}^{\Lambda} d s \Phi_{b}[\mathbf{q}(s)] e^{-i 2 \pi l s / \Lambda} .
$$

Similarly, the Fourier coefficients corresponding to the outside representation are

$$
\widetilde{\psi}_{\text {lead }, l}=\mathbb{H}_{i n l}+\sum_{j, m} S_{m n}^{(j i)} \mathbb{H}_{j m l},
$$

where

$$
\mathbb{H}_{j m l}=\frac{1}{\Lambda} \int_{0}^{\Lambda} d s \phi_{n}^{(j)}\left[y_{j}(s)\right] e^{-i 2 \pi l s / \Lambda} .
$$

Using Eq. (A5) to eliminate $S_{m n}^{(j i)}$ and equating Fourier coefficients $\widetilde{\psi}_{\text {junc }, l}=\widetilde{\psi}_{\text {lead }, l}$, we obtain a set of linear equations for the $c_{b}$ :

$$
\sum_{b}\left[\mathbb{G}_{b l}+\sum_{j, m} \mathbb{F}_{j m b} \mathbb{H}_{j m l}\right] c_{b}=2 \mathbb{H}_{i n l} .
$$

One generally chooses $N_{l}$, the number of Fourier coefficients, to be equal to $N_{b}$, the number of junction basis functions, and then solves for the $c_{b} . S_{m n}^{(j i)}$ can then be obtained using Eq. (A5). These are the desired $S$-matrix elements for the propagating modes.

Initially, we followed Ref. 31 in using plane waves traveling in different directions as a basis:

$$
\Phi_{\alpha}(\mathbf{q})=e^{i \mathbf{k}_{\alpha} \cdot \mathbf{q}}
$$

(here we have replaced the index $b$ with the angle $\alpha$ ), where

$$
\mathbf{k}_{\alpha} \cdot \mathbf{q}=k_{F} r \cos (\theta-\alpha),
$$

$r=\sqrt{x^{2}+y^{2}}, \tan (\theta)=y / x$ and $\alpha=[0 \ldots 2 \pi]$. However, we found that this basis set has poor convergence properties for the system under consideration. The dotted curves in Fig. 13 represent results obtained for the junction treated in Sec. IV with $k_{F} w=3.16738$ (one propagating mode). It is seen that the unitarity condition, $\left|S_{11}^{(21)}\right|^{2}+\left|S_{11}^{(11)}\right|^{2}=1$ is significantly violated for basis sizes larger than $N_{b} \sim 120$. However, to have enough angular resolution (with $\alpha$ chosen uniformly in its range) to describe even the first evanescent mode $(m=2$ here), $N_{b}$ must be larger than about 160 .

The cause of the instability in the plane-wave expansion for a junction of approximate circular geometry can be understood as follows. The derivative of the wave function along the perimeter is discontinuous at the edge of the leads.

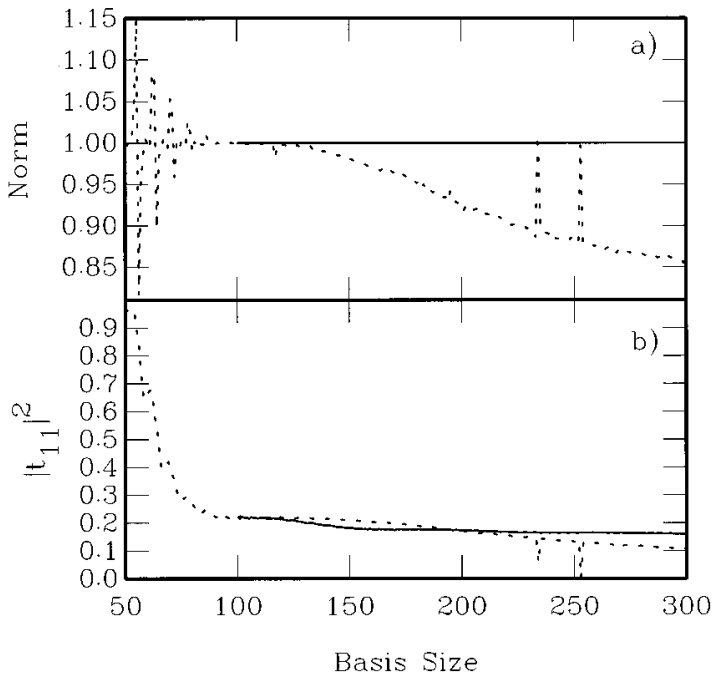

FIG. 13. Comparison of convergence properties for plane-wave (dotted curves) and Bessel-function (solid curves) basis sets as a function of basis set size. (a) Norm $(T+R)$. (b) An individual matrix element.

For this reason the Fourier summations are slowly converging (high Fourier components make important contributions). For the circular junction, the perimeter variable can be approximately written as $s \sim \theta \Lambda / 2 \pi$, so that

$$
\begin{aligned}
\mathrm{G}_{\alpha l} & \sim \frac{1}{2 \pi} \int_{0}^{2 \pi} d \theta e^{-i l \theta} e^{i k_{F} r \cos (\theta-\alpha)} \\
& =(i)^{l} J_{l}\left(k_{F} r\right) e^{-i l \alpha},
\end{aligned}
$$

where $J_{l}(x)$ is the Bessel function of the first kind. For large $l$,

$$
J_{l}(x) \rightarrow \frac{1}{\sqrt{2 \pi l}}\left(\frac{e}{2}\right)^{l}\left(\frac{x}{l}\right)^{l}
$$

so $J_{l}(x)$ becomes quite small for $x<l, l$ large. For example, $J_{200}(36) \sim 3 \times 10^{-125}$.

Because $\widetilde{\psi}_{\mathrm{junc}, l}$ must be of comparable magnitude for small and large $|l|, c_{b}$ must be quite large by Eq. (A8). But in order for $\widetilde{\psi}_{\text {junc, } l}$ to be of reasonable size for small $l$, the sum of the large terms must cancel. The result is a numerically singular matrix defined on the left-hand side of Eq. (A12).

We therefore chose to use a basis set of Bessel functions directly,

$$
\Phi_{b}(\mathbf{q})=\Gamma_{b} J_{b}\left(k_{F} r\right) e^{i b \theta}
$$

These are the solutions to the free-particle Schrödinger equation when expressed in circular coordinates. As previously noted, $J_{b}$ becomes quite small for large $b$, but in this basis set it is straightforward to directly rescale the Bessel functions with weights $\Gamma_{b}$ such that each $\Gamma_{b} J_{b}\left(k_{F} r\right)$ has a comparable magnitude around the perimeter ( $r$ is approximately constant). This basis set gives much better convergence behavior than the plane waves, as is seen in Fig. 13. Note, however, the drift of $T_{11}$ over the range of basis sizes 
$N_{b}=100 \rightarrow 300$. This is due to the slow convergence of the Fourier sum mentioned previously, so that large basis sets are necessary. In our calculations we used a basis size of 400 for one open lead mode up to 900 for four open modes. Flux was conserved to $0.03 \%$ or better for all calculations.

In Refs. 6 and 32 bases of unrescaled Bessel functions were used. In those works the numerical instability was circumvented using singular value decomposition. We also tried this method, but found that it did not work well for our case; rescaling the Bessel functions gave much better results.

This Bessel function expansion works for circular geometry. For arbitrary junction geometries, the boundary element method given in Appendix $\mathrm{C}$ is better.

\section{APPENDIX B: DIFFRACTIVE SCATTERING FROM LEAD APERTURES}

In Sec. III we approximated the wave function near the mouth of the exit lead as a sum of plane waves. Let us reexamine one of these plane waves incident on a lead mouth. While some of the amplitude associated with the plane wave can exit the junction on this encounter, a portion of the amplitude will be diffractively reflected from the narrow mouth of the lead, and will continue to bounce around the junction. This reflection produces some of the peaks seen in Fig. 8(B).

Diffractive reflection from an opening can be treated by Babinet's principle. ${ }^{26}$ The total reflected wave is the plane wave produced by reflection from a complete wall minus the wave produced by a small barrier coinciding with the opening. To apply this method, we use the following approach.

First let us note that a wave reflected from a circular wall differs in an essential way from a wave reflected from a straight-line wall. Parallel trajectories incident on a circular boundary will cross each other at a focus after reflection, while there is no focusing of like trajectories reflected from a line. If a refocusing occurs, it produces an increment in the Maslov index, so that the phase associated with the reflected wave differs by $\pi / 2$ between the two wall types. Naturally, all of our calculated reflections take this into account.

To apply Babinet's principle, we smoothly extend the boundary across the lead mouth, i.e., we continue it as an arc of a circle (not as a straight line across the lead mouth). Let us call the wave function reflected from that small circular $\operatorname{arc} \psi_{\text {arc }}^{\text {refl }}$ and the wave function reflected from the entire circular wall including the arc $\psi_{\text {circle }}^{\text {refl }}$ Then according to Babinet's principle the actual reflected wave is the difference between these two,

$$
\psi^{\text {refl }}=\psi_{\text {circle }}^{\text {refl }}-\psi_{\text {arc }}^{\text {refl }} .
$$

The wave reflected from the circle is obtained from the semiclassical approximation. This wave reflects as if the lead were not present. The wave reflected from the arc can be calculated by again applying the Kirchhoff approximation: each point on the small circular arc is a point source weighted by the incident semiclassical wave at that point. Equation (13) is used, but now the boundary is a small circular arc instead of of a straight line.

Finally, we make one more approximation. The arc is sufficiently small that we can replace it by a straight line segment. Then if the wave incident on this line segment is a

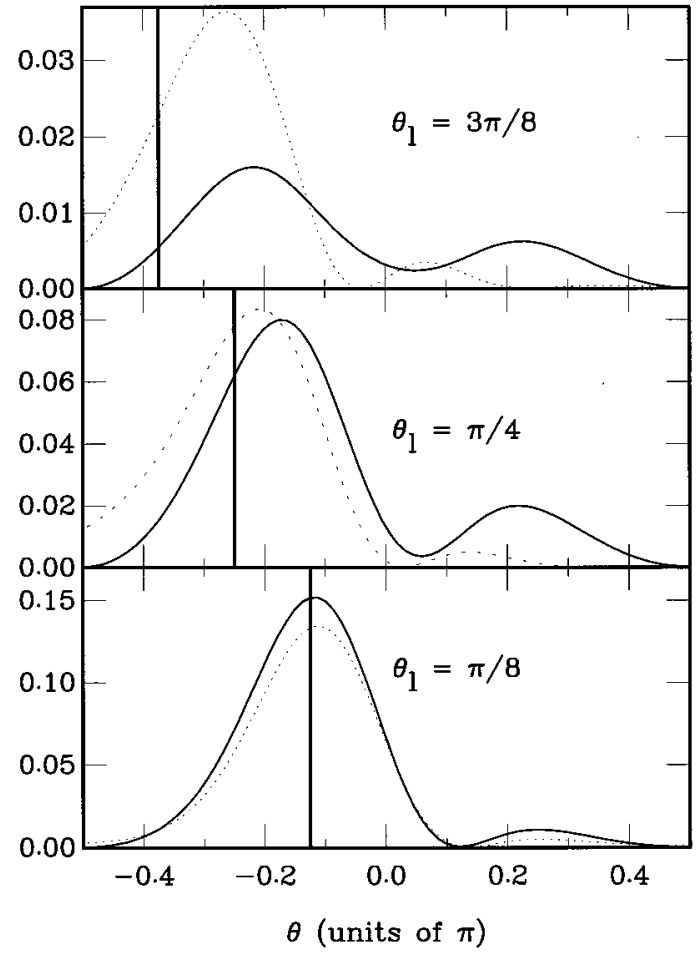

FIG. 14. $\left|\psi_{\text {line }}^{\text {refl }}(r=8 w, \theta)\right|^{2}$ for three values of $\theta_{l}$ at $k_{F}=2.5 \pi / w$ is shown as the dotted curves, from Eq. (B2). The solid curves are exact solutions of a plane wave diffracting from a lead mouth as described in Appendix C. The thick vertical bars represent the direction of the classical shadow.

plane wave $\exp \left[-i k_{F} r_{j} \cos \left(\theta_{j}-\theta_{l}\right)\right]$ moving in the direction $\theta_{l}$ of the $l$ th trajectory approaching lead $j$, the Kirchhoff formula gives

$$
\psi_{\text {line }}^{\text {refl }} \approx-\frac{\exp \left(i k_{F} r_{j}\right)}{\sqrt{r_{j}}} \Theta_{j}^{\mathrm{scat}}\left(\theta_{j} ; \theta_{l}\right)
$$

where

$$
\begin{aligned}
\Theta_{j}^{\text {scat }}\left(\theta_{j} ; \theta_{l}\right)= & -i w_{j}\left(\frac{k_{F}}{8 \pi}\right)^{1 / 2} e^{i \pi / 4}\left(\cos \theta_{j}+\cos \theta_{l}\right) \\
& \times \operatorname{sinc}\left[\frac{1}{2} k_{F} w_{j}\left(\sin \theta_{j}+\sin \theta_{l}\right)\right] \\
& \operatorname{sinc}(x)=\sin (x) / x
\end{aligned}
$$

We have an outgoing circular wave with angular dependence given by $\Theta_{j}^{\text {scat }}\left(\theta_{j} ; \theta_{l}\right)$ representing the diffractive "shadow" of the lead aperture. Plots of the modulus squared of this formula are shown in Fig. 14 for three angles at $k_{F}=2.5 \pi / w$, and compared with the exact quantum solution (of an infinite lead connected to a half-plane). The quantum solution was computed using the method of Appendix C.

Equation (B2) may seem to contradict our physical description in the third paragraph of this appendix: Eq. (B2) describes an outgoing circular wave with diverging rays, while a wave reflected from a circular arc should have converging rays. However, we have tested this approximation by calculating the exact quantum wave reflected from a circular 
arc, and found that the approximation is adequate provided that the arc is not too many wavelengths long.

Finally let us note that our use of Babinet's principle applies if the lead apertures open into "empty space" outside of the junction. We have not taken into account the fact that the leads are straight wires of fixed width. This approximation seems to be an appropriate first step, but the wire nature of the leads may be important in quantitative comparisons with quantum results.

The shadow corresponding to $\psi_{\text {line }}^{\text {refl }}$ is represented by a new family of trajectories launched at all angles a distance $r_{j}$ from the center of the lead mouth. These trajectories are connected to $\psi_{\text {line }}^{\text {refl }}$ in a manner directly analogous to the method used at the initial entrance lead. For instance, to include the lead-scattered trajectories for one and two encounters with the lead mouths one modifies Eq. (39) in the following manner: a) include those trajectories which exit once or twice before encountering lead $j$ in the sum in Eq. (39); and (b) add the components corresponding to diffractive scattering from the resulting value of the $S$-matrix element, i.e., add

$$
\begin{aligned}
e^{i \pi / 4} & \sqrt{2 \pi k_{F}} \sum_{j_{1}, j_{2}} \sum_{l_{1}, l_{3}}\left\{\Theta _ { i , n } ^ { \text { in } } ( \theta _ { i , l _ { 1 } } ) \mathcal { T } _ { l } \left[\delta_{j_{1}, j_{2}} \Theta_{j_{1}}^{\text {scat }}\left(\theta_{j_{1}, l_{1}}, \theta_{j_{2}, l_{3}}\right)\right.\right. \\
& \left.+\sum_{l_{2}} \Theta_{j_{1}}^{\text {scat }}\left(\theta_{j_{1}, l_{1}}, \theta_{j_{1}, l_{2}}\right) \mathcal{T}_{l} \Theta_{j_{2}}^{\text {scat }}\left(\theta_{j_{2}, l_{2}}, \theta_{j_{2}, l_{3}}\right)\right] \\
& \left.\times \mathcal{T}_{l} \Theta_{j, m}^{\text {in }}\left(\theta_{j_{2}, l_{3}}\right)\right\},
\end{aligned}
$$

where $j_{1}$ and $j_{2}$ label intermediate leads and $l_{1}, l_{2}$, and $l_{3}$ label trajectories between leads $i$ and $j_{1}, j_{1}$ and $j_{2}$, and $j_{2}$ and $j$, respectively.

To correctly achieve unitarity in the relatively large wavelength to lead-width regime studied here, the diffractive scattering process must conserve flux. In particular, we require that the flux integral Eq. (11) is obeyed for the contour shown in Fig. 3, where $\psi_{1}=\psi_{2}=\psi_{\text {lead }}$ for interval $A B$, and $\psi_{1}=\psi_{2}=\psi_{\text {inc }}+\psi_{\text {ref }}-\psi_{\text {shadow }}$ for arc $C D$. The approximation which we used for $\psi_{\text {shadow }}$, Eq. (B2), does not obey this current conservation condition, so strict unitarity should not be expected here, even if a very large number of trajectories were included.

\section{APPENDIX C: SOLUTION OF BILLIARD SCATTERING PROBLEMS USING THE BOUNDARY ELEMENT METHOD}

The boundary element method (BEM) or boundary integral method has been widely used in engineering ${ }^{33}$ and physics. ${ }^{34,35} \mathrm{We}$ use it to solve quantum scattering problems for billiardlike potentials.

The starting point for the BEM is the KirchhoffHelmholtz equation, which expresses the wave function at point $\mathbf{q}$ in terms of the wave function and its normal derivative on a contour $\mathcal{C}$ enclosing region $\mathcal{A}$.

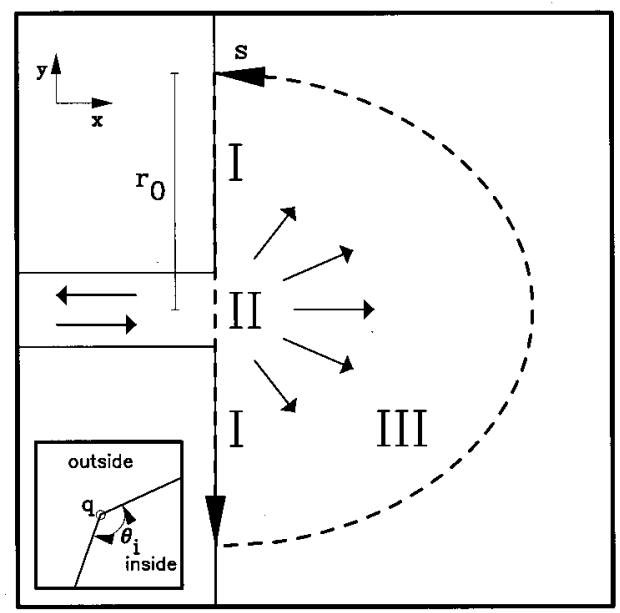

FIG. 15. Boundary contour definition for diffraction at the interface between a lead and a half-plane. Inset: definition of $\theta_{i}$.

$$
\begin{aligned}
\frac{\theta_{i}}{2 \pi} \psi(\mathbf{q})= & \oint_{\mathcal{C}} d s^{\prime}\left[\psi\left[\mathbf{q}^{\prime}\left(s^{\prime}\right)\right] \frac{\partial}{\partial n^{\prime}} G\left[\mathbf{q}, \mathbf{q}^{\prime}\left(s^{\prime}\right)\right]\right. \\
& \left.-G\left[\mathbf{q}, \mathbf{q}^{\prime}\left(s^{\prime}\right)\right] \frac{\partial}{\partial n^{\prime}} \psi\left[\mathbf{q}^{\prime}\left(s^{\prime}\right)\right]\right] \\
& +4 i \frac{\mu e}{\hbar c} \oint_{\mathcal{C}} d s^{\prime} G\left[\mathbf{q}, \mathbf{q}^{\prime}\left(s^{\prime}\right)\right] \psi\left[\mathbf{q}^{\prime}\left(s^{\prime}\right)\right] \\
& \times \mathbf{A}\left[\mathbf{q}^{\prime}\left(s^{\prime}\right)\right] \cdot \hat{\mathbf{n}}^{\prime},
\end{aligned}
$$

where $G\left(\mathbf{q}, \mathbf{q}^{\prime}\right)$ is the free-particle Green function, and $\hat{\mathbf{n}}^{\prime}$ is the inward-pointing normal on the contour. $\mathbf{A}$ is the vector potential, which is taken to be zero in the current study. $\theta_{i}$ is called the internal angle. For $\mathbf{q}$ inside the region $\mathcal{A}$, $\theta_{i}=2 \pi$. For $\mathbf{q}$ outside this region, $\theta_{i}=0$. On the boundary $\theta_{i}$ is defined in the inset of Fig. 15. At any point at which the boundary is a smooth curve, $\theta_{i}=\pi$, while if the boundary has a sharp bend, $\theta_{i}$ for the vertex is the angle subtended inside region $\mathcal{A}$.

In the BEM the bounding contour $\mathcal{C}$ is discretized, and the wave function and its normal derivative on this curve are solved for. Once values are known on the surface, the wave function anywhere in $\mathcal{A}$ can be calculated using Eq. (C1).

Let us introduce some simplifying notation. For points $\mathbf{q}$ and $\mathbf{q}^{\prime}$ on the boundary,

$$
\begin{gathered}
g\left(s^{\prime}\right) \equiv \psi\left[\mathbf{q}^{\prime}\left(s^{\prime}\right)\right], \\
\left.f\left(s^{\prime}\right) \equiv \frac{\partial}{\partial n^{\prime}} \psi\left[\mathbf{q}^{\prime}\right]\right|_{\mathbf{q}^{\prime}=\mathbf{q}^{\prime}\left(s^{\prime}\right)}, \\
G\left(s, s^{\prime}\right) \equiv G\left[\mathbf{q}(s), \mathbf{q}^{\prime}\left(s^{\prime}\right)\right], \\
\left.\dot{G}\left(s, s^{\prime}\right) \equiv \frac{\partial}{\partial n^{\prime}} G\left[\mathbf{q}(s), \mathbf{q}^{\prime}\right]\right|_{\mathbf{q}^{\prime}=\mathbf{q}^{\prime}\left(s^{\prime}\right)} .
\end{gathered}
$$

Then, for smooth boundaries and $\mathbf{A}=\mathbf{0}$, we can rewrite Eq. (C1) as 
$\frac{1}{2} g(s)=\oint_{\mathcal{C}} d s^{\prime}\left[\dot{G}\left(s, s^{\prime}\right) g\left(s^{\prime}\right)-G\left(s, s^{\prime}\right) f\left(s^{\prime}\right)\right]$.

We wish for $\psi$ to obey boundary conditions, corresponding to physical constraints. These boundary conditions imply that the value of a wave function and the value of its normal derivative on the boundary are not independent. For hardwall boundary conditions it is convenient to write these constraints in a general form:

$$
g(s)=N(s)+\oint_{\mathcal{C}} d s^{\prime} M\left(s, s^{\prime}\right) f\left(s^{\prime}\right) .
$$

$N(s)$ and $M\left(s, s^{\prime}\right)$ will be specified later, as particular cases are considered. For the moment, we are just asserting that the boundary conditions imply a second linear integral relation between $f\left(s^{\prime}\right)$ and $g(s)$.

For $G\left(\mathbf{q}, \mathbf{q}^{\prime}\right)$ it is convenient to use the point source Green function, which for zero magnetic field is given by Eq. (14) (the point-source Green function for nonzero magnetic field is also known ${ }^{36}$ ).

Substituting Eq. (C7) into Eq. (C6) and rearranging gives an inhomogeneous linear integral equation for $f(s)$ :

$$
\oint_{\mathcal{C}} d s^{\prime} K\left(s, s^{\prime}\right) f\left(s^{\prime}\right)=I(s)
$$

where

$$
\begin{aligned}
K\left(s, s^{\prime}\right)= & -\frac{1}{2} M\left(s, s^{\prime}\right)+\oint_{\mathcal{C}} d s^{\prime \prime} \dot{G}\left(s, s^{\prime \prime}\right) M\left(s^{\prime \prime}, s^{\prime}\right) \\
& -G\left(s, s^{\prime}\right)
\end{aligned}
$$

and

$$
I(s)=\frac{1}{2} N(s)-\oint_{\mathcal{C}} d s^{\prime} \dot{G}\left(s, s^{\prime}\right) N\left(s^{\prime}\right) .
$$

We now apply the above procedure to two scattering problems involving the geometry shown in Fig. 15.

\section{Incoming lead state diffracting into an infinite half-plane}

Consider a single incoming lead state propagating in the positive- $x$ direction. It diffracts at $x=0$, part of the wave function is scattered back into the lead, and some becomes an outgoing circular wave (with some angular modulation) in the half-plane $x>0$.

The full wave function in the lead can be written

$$
\psi^{\text {lead }}(\mathbf{q} ; n)=\psi_{n}^{\text {lead,in }}(\mathbf{q})+\sum_{m=1}^{\infty} c_{m} \psi_{m}^{\text {lead,out }}(\mathbf{q}),
$$

where $\psi_{n}^{\text {lead,in }}$ and $\psi_{m}^{\text {lead,out }}$ are defined as in Sec. III A. The coefficients $c_{m}$ are unknown.

In the half-plane, for $r>w / 2$, the wave function can be expanded in a basis of outgoing Hankel functions with angular components which satisfy the hard-wall boundary conditions

$$
\psi^{\mathrm{out}}=\sum_{n=1}^{\infty} d_{n} \sin [n(\theta+\pi / 2)] H_{n}^{(1)}\left(k_{F} r\right) / H_{n}^{(1)}\left(k_{F} r_{0}\right)
$$

with coefficients $d_{n}$. The $H_{n}^{(1)}\left(k_{F} r_{0}\right)$ has been included in the denominator to ensure that the $d_{n}$ are of the same order of magnitude, as was done in Appendix A.

Figure 15 defines a bounding contour on which we solve for $f(s)$. There are three distinct intervals on the contour, in which the wave function must satisfy different boundary conditions. In interval I, the boundary lies along a wall so that the wave function is zero. Interval II spans the mouth of the lead, while interval III traces out an arc of constant $r=r_{0}$ in the half-plane.

In interval II, the boundary lies along $x=0$, $-w / 2<y<w / 2$. Here we require continuity of the wave function and its normal derivative. We substitute Eqs. (3) and (5) into Eq. (C11) and take the $x$ derivative to obtain

$$
f(s)=i \frac{k_{n}}{\sqrt{v_{n}}} \phi_{n}[y(s)]-i \sum_{m=1}^{\infty} c_{m} \frac{k_{m}}{\sqrt{v_{m}}} \phi_{m}[y(s)] .
$$

Because of the orthogonality of the $\phi_{m}$, it is convenient to multiply this equation by $\phi_{m}(y)$ and integrate over the lead mouth to get an expression for the $c_{m}$ in terms of $f(s)$, in analogy to Eq. (A5). Now, for points in the lead mouth we combine this expression with Eqs. (C11), (3), and (5) to obtain

$$
\begin{aligned}
g(s)= & \phi_{n}[y(s)] / \sqrt{v_{n}}+\sum_{m} c_{m} \phi_{m}[y(s)] / \sqrt{v_{m}} \\
= & 2 \phi_{n}[y(s)] / \sqrt{v_{n}}+\int d s^{\prime}\left(\frac{d y}{d s^{\prime}}\right) \sum_{m} \frac{i}{k_{m}} \phi_{m}[y(s)] \\
& \times \phi_{m}\left[y\left(s^{\prime}\right)\right] f\left(s^{\prime}\right),
\end{aligned}
$$

where the integral is over the lead mouth. This has the form of Eq. (C7); $N(s)$ and $M\left(s, s^{\prime}\right)$ can be read in Eq. (C14).

On the arc (interval III), the inward normal is in the $-r$ direction, so

$f(s)=-\sum_{n=1}^{\infty} d_{n} \sin [n(\theta(s)+\pi / 2)] \frac{\partial}{\partial r} H_{n}^{(1)}\left(k_{F} r\right) / H_{n}^{(1)}\left(k_{F} r_{0}\right)$

Again, the $\theta$-dependent functions are orthogonal, so the $d_{n}$ can be expressed in terms of $f(s)$. The wave function on the arc is then

$$
\begin{aligned}
g(s)= & \sum_{n} d_{n} \sin [n(\theta(s)+\pi / 2)] \\
= & \int d s^{\prime} \sum_{n}\left(-\frac{2}{\pi k_{F}}\right) \frac{H_{n}^{(1)}\left(k_{F} r_{0}\right)}{H_{n}^{(1) \prime}\left(k_{F} r\right)}\left(\frac{d \theta}{d s^{\prime}}\right) \\
& \times \sin \left[n\left(\theta(s)+\frac{\pi}{2}\right)\right] \sin \left[n\left(\theta\left(s^{\prime}\right)+\frac{\pi}{2}\right)\right] f\left[\theta\left(s^{\prime}\right)\right] .
\end{aligned}
$$




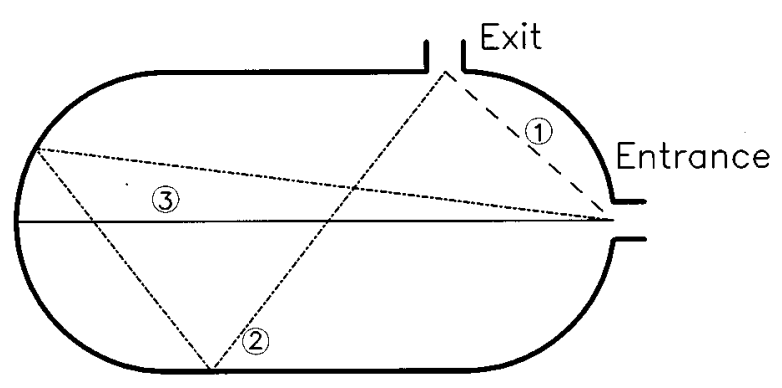

FIG. 16. Geometry of the stadium. The straight wall segments are of length 2 and the radius of the circular ends is 1 . The lead mouths both have width $0.0935 \sqrt{\pi+4}$. Also shown are some important trajectories.

We have again arrived at a form like Eq. (C7), with $N(s)=0$ and $M\left(s, s^{\prime}\right)$, the kernel of the integral in Eq. (C16). This method was used to obtain the quantum results in Fig. 4.

\section{Incoming plane wave diffracting off a lead mouth}

Here we consider the same geometry as in the previous case, but we change the incoming asymptotic conditions to be a plane wave in the half-plane incident on the lead mouth at an angle $\alpha$. In the half-plane we write the wave function as a sum of incident, reflected, and diffracted parts,

$$
\psi_{h p}=\psi_{\text {inc }}+\psi_{\text {ref }}-\psi_{d},
$$

where

$$
\begin{aligned}
& \psi_{\mathrm{inc}}=e^{-i k_{F}[x \cos \alpha+y \sin \alpha]}, \\
& \psi_{\mathrm{ref}}=-e^{i k_{F}[x \cos \alpha-y \sin \alpha]} .
\end{aligned}
$$

$\psi_{d}$ is to be determined. In the lead

$$
\psi_{\text {lead }}=\sum_{m=1}^{\infty} c_{m} \psi_{m}^{\text {lead,out }}
$$

In the lead mouth we require that the total wave function and its normal derivative be continuous,

$$
\psi_{\text {lead }}[x=0, y(s)]=\psi_{\mathrm{hp}}=-\psi_{d}=g(s)
$$

and

$$
\frac{\partial}{\partial x} \psi_{\text {lead }}=\frac{\partial}{\partial x} \psi_{\mathrm{hp}}=-2 i k_{F} \cos (\alpha) e^{-i k_{F} y(s) \sin \alpha}+f(s) .
$$
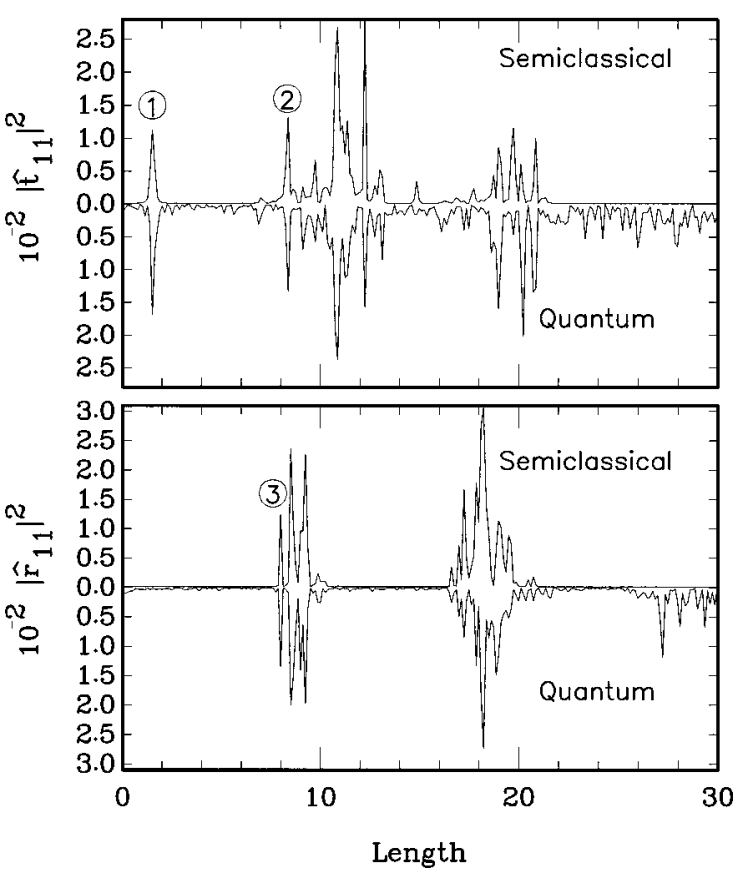

FIG. 17. Power spectrum of the transmission and reflection amplitudes for the stadium. Peaks associated with some short paths are numbered.

A procedure analogous to that used in the previous example can then be used to obtain a linear equation for $f(s)$. The quantum results in Fig. 14 were obtained using this method.

\section{APPENDIX D: A STADIUM}

We mentioned that the semiclassical formula is easily applied to any geometry. The BEM can also be used for a variety of geometries. We carried out quantum and semiclassical calculations of the transmission and reflection amplitudes for a stadium geometry. Figure 16 shows the geometry of the stadium and a few important trajectories. Figure 17 shows the power spectra of the transmission and reflection amplitudes. In the semiclassical calculation we included only paths with no more than eight bounces, and we neglected diffractive scattering. Again, the semiclassical formula gives a good approximation.

We made no attempt to optimize the efficiency of the computer codes for either calculation. For a crude comparison, let us remark that the quantum calculation took almost three orders of magnitude more computer time than its semiclassical counterpart.
${ }^{1}$ C.M. Marcus, A.J. Rimberg, R.M. Westervelt, P.F. Hopkins, and A.C. Gossard, Phys. Rev. Lett. 69, 506 (1992); C.M. Marcus, R.M. Westervelt, P.F. Hopkins and A.C. Gossard, Chaos 3, 643 (1993); Surf. Sci. 305, 480 (1994).

${ }^{2}$ M.W. Keller, O. Millo, A. Mittal, D.E. Prober, and R.N. Sacks, Surf. Sci. 305, 501 (1994).

${ }^{3}$ H.U. Baranger and P.A. Mello, Phys. Rev. Lett. 73, 142 (1994).

${ }^{4}$ R.A. Jalabert, J.-L. Pichard, and C.W.J. Beenakker, Europhys. Lett. 27, 255 (1994).
${ }^{5}$ P.W. Brouwer and C.W.J. Beenakker, Phys. Rev. B 50, 11263 (1994); 51, 7739 (1995).

${ }^{6}$ X. Xang, H., Ishio, and J., Burgdörfer, Phys. Rev. B 52, 8219 (1995).

${ }^{7}$ For example, see B.L. Al'tshuler, Pis'ma Zh. Éksp. Teor. Fiz. 41, 530 (1985), [JETP Lett. 41, 648 (1985)]; B. L. Al'tshuler and B. I. Shklovskiǔ, Zh. Éksp. Teor. Fiz. 91, 220 (1986) [Sov. Phys. JETP 64, 127 (1986)].

${ }^{8}$ R.V. Jensen, Chaos 1, 101 (1991); W.A. Lin, J.B. Delos, and 
R.V. Jensen, ibid. 3, 655 (1993).

${ }^{9}$ N. Argaman, Phys. Rev. Lett. 75, 2750 (1995).

${ }^{10}$ H.U. Baranger, D.P. DiVincenzo, R.A. Jalabert, and A.D. Stone, Phys. Rev. B 44, 10637 (1991).

${ }^{11}$ R.A. Jalabert, H.U. Baranger, and A.D. Stone, Phys. Rev. Lett. 65, 2442 (1990); H.U. Baranger, R.A. Jalabert, and A.D. Stone, Chaos 3, 665 (1993).

${ }^{12}$ Y. Avishai, J. Bar Touv, Y.B. Band, and M. Kaveh, Physica A 168, 433 (1990).

${ }^{13}$ H. Ishio and J. Burgdörfer, Phys. Rev. B 51, 2013 (1995).

${ }^{14}$ Y. Wang, J. Wang, and H. Guo, Phys. Rev. B 49, 1928 (1994).

${ }^{15}$ K. Nakamura, K. Ito, and Y. Takane, J. Phys. A 27, 5889 (1994).

${ }^{16}$ W.A. Lin and R.V. Jensen, Phys. Rev. B (to be published).

${ }^{17}$ D. Weiss, K. Richter, A. Menschig, R. Bergmann, H. Schweizer, K. von Klitzing, and G. Weimann, Phys. Rev. Lett. 70, 4118 (1993); K. Richter, Europhys. Lett. 29, 7 (1995).

${ }^{18}$ G. Hackenbroich and F. von Oppen, Europhys. Lett. 29, 151 (1995).

${ }^{19}$ J. B. Keller, J. Appl. Phys. 28, 426 (1957).

${ }^{20}$ G. Vattay, A. Wirzba, and P.E. Rosenqvist, Phys. Rev. Lett. 73, 2304 (1994).

${ }^{21}$ R. Landauer, IBM J. Res. Dev. 1, 223 (1957); Philos. Mag. 21, 863 (1970).

${ }^{22}$ M. Büttiker, Phys. Rev. Lett. 57, 1761 (1986).

${ }^{23}$ H.U. Baranger and A.D. Stone, Phys. Rev. B 40, 8169 (1989).

${ }^{24}$ J.B. Delos, J.A. Alford, W.A. Lin, and R.V. Jensen in Chaos in Mesoscopic Systems, Proceedings of the Mini-workshop on Nonlinearity and the Adiriatico Research Conference on Mesoscopic Systems and Chaos: A Novel Approach, edited by G.
Casati and H. A. Cerdeira (World Scientific, Singapore, 1995), pp. $151-158$.

${ }^{25}$ A. Messiah, Quantum Mechanics (North-Holland, Amsterdam, 1961), p. 372.

${ }^{26}$ J.D. Jackson, Classical Electrodynamics (Wiley, New York, 1975), p. 427.

${ }^{27}$ P.M. Morse and H. Feshbach, Methods of Theoretical Physics (McGraw-Hill, New York, 1953), Vol. 1, p. 811.

${ }^{28}$ For hard-wall stadia, this derivative can also be expressed as a product of factors associated with path segments between each bounce [W.A. Lin (unpublished)].

${ }^{29}$ We have also carried out some calculations with more refined approximations for $\psi_{j, m}^{\text {out,diff }}$ beyond the Kirchhoff approximation. For those calculations, it was important to integrate on the arc rather than across the lead mouth.

${ }^{30}$ To avoid spurious results due to the singularity at threshold in our calculation of the power spectrum, we excluded a region near threshold: as in the quantum calculations, we evaluated $S_{11}^{(j i)}$ at 797 discrete points in the range $w k_{F} / \pi=[1.01 \ldots 5.01]$. In calculating power spectra, we did not include the first 19 data points.

${ }^{31}$ K. Nakamura and H. Ishio, J. Phys. Soc. Jpn. 61, 3939 (1992).

${ }^{32}$ E. Doron and U. Smilansky, Physica D 50, 367 (1991).

${ }^{33}$ C.A. Brebbia, J.C.F. Telles, and L.C. Wrobel, Boundary Element Techniques (Springer-Verlag, Berlin, 1984).

${ }^{34}$ M.V. Berry and M. Wilkinson, Proc. R. Soc. London Ser. A 392, 15 (1984).

${ }^{35} \mathrm{~B}$. Li and M. Robnik (unpublished).

${ }^{36}$ T. Ueta, J. Phys. Soc. Jpn. 61, 4314 (1992). 